\title{
Activation of NRF2/FPN1 pathway attenuates myocardial ischemia-reperfusion injury in diabetic rats by regulating iron homeostasis and ferroptosis
}

\author{
Hao Tian ${ }^{1} \cdot$ Yonghong Xiong ${ }^{1} \cdot$ Yi Zhang $^{2} \cdot$ Yan Leng $^{1} \cdot$ Jie Tao $^{1} \cdot$ Lu Li $^{1} \cdot$ Zhen Qiu $^{1} \cdot$ Zhongyuan Xia $^{1}$ (I)
}

Received: 1 December 2021 / Revised: 16 January 2022 / Accepted: 26 January 2022 / Published online: 5 February 2022

(c) The Author(s) 2022

\begin{abstract}
In patients with ischemic heart disease, myocardial ischemia-reperfusion injury (IRI) can aggravate their condition even worse, and diabetes increases their risk of myocardial IRI. Pathological pathways of common diseases and surgical operations like diabetes, obesity, coronary artery angioplasty, and heart transplantation entail disorders of iron metabolism. Ferroportin1 (FPN1) is the only mammalian protein associated with iron release and thus plays a vital role in iron homeostasis, while nuclear factor E2-related factor 2 (NRF2) controls the transcription of FPN1. Since the NRF2/FPN1 pathway may play a favorable role in the therapy of diabetic myocardial IRI, this work investigated the possible mechanism. In this study, we investigated the effects of ferroptosis in STZ-induced diabetic rats following myocardial IRI in vivo, and its alteration in glucose and hypoxia/reoxygenation-induced cardiomyocytes injury in vitro. Rats and $\mathrm{H} 9 \mathrm{c} 2$ cardiomyocytes were randomly divided into 6 groups and treated with sulforaphane and erastin besides the establishment of diabetic myocardial IRI and hyperglycemic hypoxia-reoxygenation models. Cardiac functional and structural damage were detected by Evans blue/TTC double staining, echocardiography, HE staining, and serological indices. CCK-8 assay and ROS production were used to measure cardiomyocyte viability and oxidative stress level. Additionally, the changes in cell supernatant levels of $\mathrm{Fe}^{2+}$, SOD, MDA, and mRNA and protein expression of ferroptosis marker proteins confirmed the beneficial effects of the NRF2/FPN1 pathway on diabetic myocardial IRI related to iron metabolism and ferroptosis. Overall, these findings suggest that iron homeostasis-related ferroptosis plays an important role in aggravating myocardial IRI in diabetic rats, and NRF2/FPN1 pathway-mediated iron homeostasis and ferroptosis might be a promising therapeutic target against myocardial IRI in diabetes.
\end{abstract}

Keywords NRF2 $\cdot$ FPN1 $\cdot$ Iron homeostasis $\cdot$ Ferroptosis $\cdot$ Diabetes $\cdot$ Myocardial ischemia-reperfusion injury

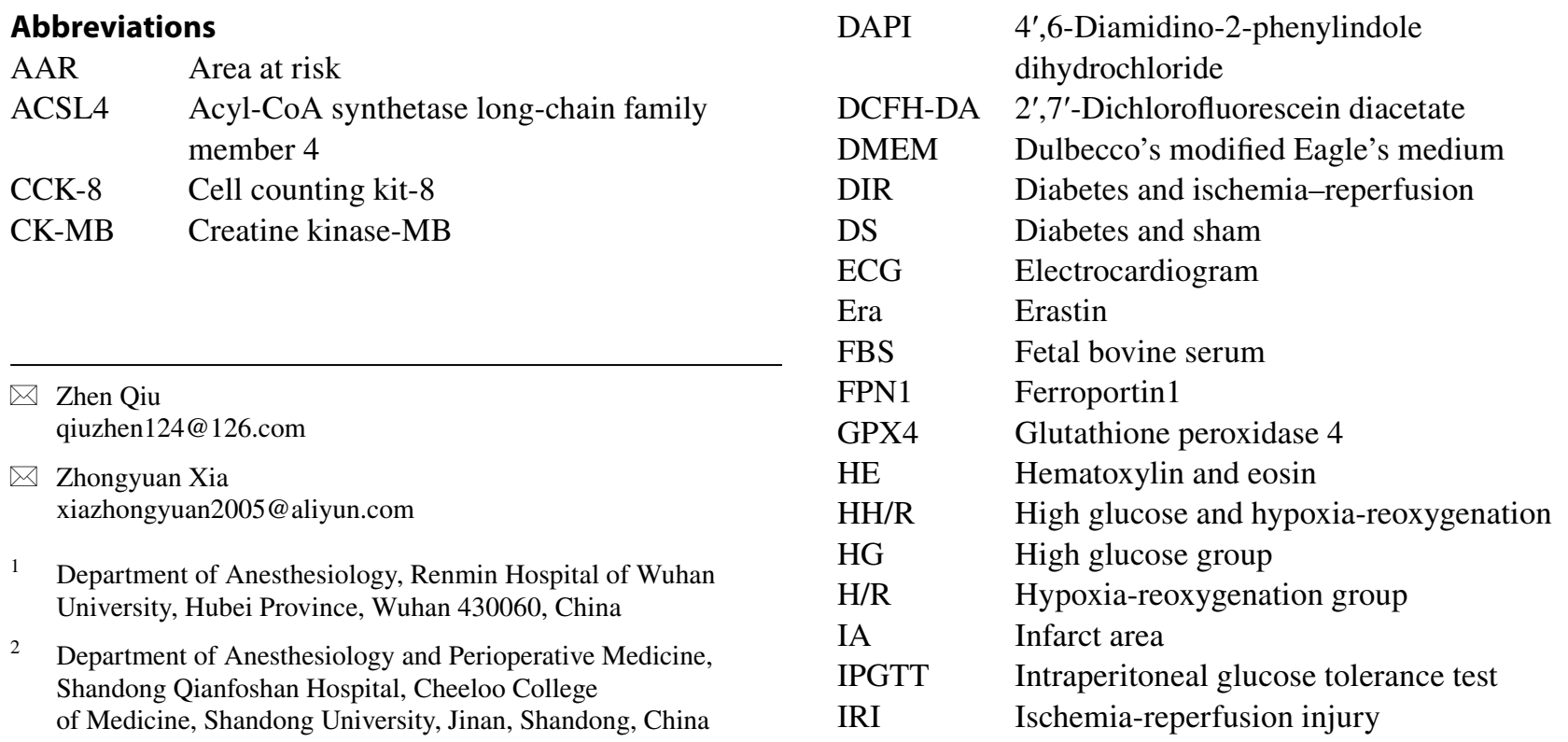




$\begin{array}{ll}\text { LAD } & \text { Left anterior descending coronary artery } \\ \text { LDH } & \text { Lactate dehydrogenase } \\ \text { LVEF } & \text { Left ventricular ejection fraction } \\ \text { LVFS } & \text { Left ventricular shortening fraction } \\ \text { MDA } & \text { Malondialdehyde } \\ \text { NIR } & \text { Normal and ischemia-reperfusion } \\ \text { NRF2 } & \text { Nuclear factor E2-related factor 2 } \\ \text { NS } & \text { Normal and sham } \\ \text { OGTT } & \text { Oral glucose tolerance test } \\ \text { ROS } & \text { Reactive oxygen species } \\ \text { SFN } & \text { Sulforaphane } \\ \text { SOD } & \text { Superoxide dismutase } \\ \text { STZ } & \text { Streptozotocin } \\ \text { TTC } & \text { Triphenyl tetrazolium chloride }\end{array}$

\section{Introduction}

Diabetes is a chronic metabolic disease marked by high blood sugar levels, and its prevalence and disability rates have risen dramatically in recent years. Persistent hyperglycemia, especially in the heart and blood vessels, can induce chronic damage and dysfunction of many tissues. Diabetic people are at risk of developing ischemic heart disease, particularly acute myocardial infarction. Coronary blood flow must be restored in order to rescue the ischemic myocardial (Crisafulli et al. 2020). However, ischemia-reperfusion injury (IRI) can occur during this process, resulting in worsened structural and functional damage (Davidson et al. 2019). Diabetes, according to prior study, can worsen myocardial IRI by causing oxidative stress via associated pathways that trigger apoptosis, inflammation, autophagy, and so on (Gan et al. 2020; Qu et al. 2019; Wang et al. 2017). However, the main related mechanisms of myocardial IRI in diabetes need to be further clarified.

Ferroptosis is a recently recognized form of iron-dependent programmed cell death that based on the inactivation of the lipid repair enzyme glutathione peroxidase 4 (GPX4) and subsequent accumulation of reactive oxygen species (ROS) in lipids. Iron chelators, such as desferrioxamine, have been demonstrated in studies to greatly reduce erastin-induced cellular ferroptosis. This indicates that iron homeostasis is crucial in the development of cellular ferroptosis (Dixon et al. 2012). Other studies have found that polyunsaturated fatty acid-containing cell and plasma membranes are particularly vulnerable to lipid radical peroxidation, and that the pace of this reaction is considerably accelerated in the presence of iron (Galluzzi et al. 2012). Thus, irons are required for the accumulation of lipid peroxide and the commencement of the ferroptosis process.

Iron is an indispensable trace metal element in a variety of physiological activities. Both excess and deficiency of iron are potential risk factors. Imbalances in iron homeostasis have been reported to be involved in a variety of diseases such as cancer, anemia, neurodegenerative diseases, and heart disease (Gozzelino and Arosio 2016). Iron uptake and iron release are key steps in the regulation of cellular iron metabolism. It is well known that the occurrence of disorders of iron metabolism in the body is associated with abnormalities of hepcidin. And ferroportin1 (FPN1) internalization and degradation mediated by hepcidin are essential in the maintenance of cardiac iron homeostasis (Lakhal-Littleton et al. 2015). Multiple iron input mechanisms exist in cardiomyocytes. But FPN1 is the only iron release-related protein found in mammals and plays a critical role in systemic iron homeostasis. FPN1 is a transmembrane protein that is responsible for iron export from the cell to the plasma and is encoded by the SLC40A1 gene (Lakhal-Littleton et al. 2015).

Nuclear factor E2-related factor 2 (NRF2), known as a critical regulator of the antioxidant response, is a transcription factor encoded by the NFE2L2 gene and belongs to the leucine zipper structure family (Moi et al. 1994). Oxidative stress plays an important role in the process of diabetes and its complications (Brownlee 2005). And oxidative stress is considered as one of the main causes of cardiac dysfunction during myocardial ischemia-reperfusion (Ichihara 2013). Zhang et al. have demonstrated that myocardial oxidative damage and cell death could be ameliorated by activating NRF2associated signaling pathways, and thus the myocardial IRI of T1D was alleviated (Zhang et al. 2018). And in the nucleus, NRF2 acts as a transcription factor to regulate iron metabolism-related gene SLC40A1 (Harada et al. 2011). Of note, iron regulatory proteins (IRPs) can recognize and bind iron responsive elements (IREs) present in the $5^{\prime}$-untranslated or in the 3 '-untranslated regions of the mRNAs encoding proteins involved in iron metabolism (Iwai 2019). Therefore, the regulation of iron homeostasis is not limited to the transcriptional level but can also be obtained through post-transcriptional regulation. Research has reported that the expression of FPN1 was decreased in NRF2 knockout mice (Liu et al. 2020a). Furthermore, studies have found that myocardium could be protected from ferroptosis by regulating NRF2 expression (La Rosa et al. 2021; Luo et al. 2021). However, the NRF2-FPN1 interaction relationship in diabetic myocardial IRI has not been reported. Therefore, the purpose of our present study aims to test the hypotheses that NRF2/FPN1 signaling pathway-mediated iron homeostasis and ferroptosis play a crucial role in diabetic myocardial IRI, which may be a protective mechanism against diabetic myocardial IRI.

\section{Materials and methods}

\section{Animals and diabetes induction}

The animal experimental protocol was approved by the Bioethics Committee of Renmin Hospital of Wuhan 
University (Animal Welfare No.20200303). Healthy male adult Sprague-Dawley (SD) rats were supplied by Beijing Vital River Bioscience Co. Ltd, weighing 200-220 g, 6-8 weeks old. After 5 days of acclimatization, the rats were fasted for $12 \mathrm{~h}$ for diabetes induction. The diabetic rats were administered a single intraperitoneal injection of $60 \mathrm{mg} / \mathrm{kg}$ streptozotocin (STZ) dissolved in citrate buffer to induce diabetes as described previously (Qiu et al. 2017). The non-diabetic rats were injected with an equal volume of sodium citrate buffer. After $72 \mathrm{~h}$ (with $6 \mathrm{~h}$ fasting), the rats exhibiting hyperglycemia (blood glucose level higher than $16.7 \mathrm{mmol} / \mathrm{l}$ ), polyphagia, polydipsia, and polyuria were considered to be suffering from diabetes.

\section{Reagents}

Streptozotocin (STZ), triphenyl tetrazolium chloride (TTC), and Evans blue (EB) were purchased from Sigma Chemical Co. (MO, USA). Dulbecco's modified Eagle's medium (DMEM) and fetal bovine serum (FBS) were obtained from Gibco Laboratories (Grand Island, NY, USA). Sulforaphane (SFN), the NRF2 activator, was purchased from Glpbio Co. (CA, USA). Erastin (Era), the ferroptosis inducer, was purchased from Selleck (Houston, TX). The cell counting kit-8 (CCK-8) and 2',7'-dichlorofluorescein diacetate (DCFH-DA) assay test kits were obtained from Beyotime Institute of Biotechnology (Shanghai, China). Lactate dehydrogenase (LDH), creatine kinase-MB (CK-MB), superoxide dismutase (SOD), and malondialdehyde (MDA) assay test kits were purchased from Nanjing Jiancheng Bioengineering Institute (Nanjing, Jiangsu, China). $\mathrm{Fe}^{2+}$ assay test kit was purchased from Abcam Co. (Abcam, UK). The primers for NRF2, FPN1, ACSL4, and $\beta$-actin were designed and synthesized by Wuhan Servicebio Co. (Wuhan, Hubei, China). NRF2 primary antibodies were purchased from Cell Signaling Technology (CST, Beverly, CA, USA). FPN1, ACSL4, GPX4, and GAPDH primary antibodies were obtained from Proteintech Co. (Wuhan, Hubei, China).

\section{Glucose tolerance testing}

The diabetic rats were fasted overnight, and then glucose was administered at a dose of $2 \mathrm{~g} / \mathrm{kg}$ by gastric lavage or intraperitoneally injection to conduct the oral glucose tolerance test (OGTT) and intraperitoneal glucose tolerance test (IPGTT) to confirm the success of animal model. Blood glucose level was measured at 0 (before glucose load), 30, 60, 90, and $120 \mathrm{~min}$. All blood samples were collected from the caudal vein to determine plasma glucose.

\section{Myocardial ischemia and reperfusion model}

Rats were anesthetized by intraperitoneal injection of $1 \%$ sodium pentobarbital $(60 \mathrm{mg} / \mathrm{kg})$ and managed for electrocardiogram (ECG) monitoring. After disinfection of skin, tracheotomy was performed and the ventilator was connected for mechanical ventilation. The ribs were cut at the 3rd to 4th intercostal space in the left midclavicular line and the entire heart was fully exposed. Subsequently, the left anterior descending coronary artery (LAD) below the left auricle was ligated with a 7-0 silk wire. Successful ischemia was indicated when there was a change in color from red to white in the apical region and left ventricular wall with reduced ventricular wall motion and an elevated ST-segment arch in the ECG. After $30 \mathrm{~min}$, the apical and left ventricular color gradually returned to red and the ST-segment recovered, suggesting successful reperfusion, as seen by loosening the ligature. Lastly, the myocardium was reperfused for $2 \mathrm{~h}$ (for protein expression and serological indicators measurement) or $72 \mathrm{~h}$ (for cardiac function measurement). Furthermore, the ligation thread was passed through the LAD but not ligated in the sham operation group.

\section{Experimental protocols}

For the in vivo study, 8 weeks after STZ injection, all rats were randomly divided into 4 groups: (1) normal + sham group (NS); (2) normal + ischemia-reperfusion group (NIR); (3) diabetes + sham group (DS); (4) diabetes + ischemia-reperfusion group (DIR). To gain a deeper insight into the effect of NRF2 activation, the following experiments were preformed among diabetic rats: (1) diabetes + ischemia-reperfusion group (DIR); (2) diabetes + ischemia-reperfusion + sulforaphane group $(\mathrm{DIR}+\mathrm{SFN})$; (3) diabetes + ischemia-reperfusion $+\mathrm{SFN}+$ erastin group $($ DIR + SFN + Era). Each group contains 8 rats. The NRF2 activator sulforaphane $(500 \mu \mathrm{g} / \mathrm{kg} /$ day $)$ was injected intraperitoneally for 3 days before ischemia (Piao et al. 2010). And the ferroptosis inducer Erastin $(20 \mathrm{mg} / \mathrm{kg})$ was injected intraperitoneally at the beginning of the ischemia-reperfusion operation.

For the in vitro study, H9c2 cardiomyocytes, a subclonal cell line derived from embryonic BD1X rat myocardial tissue, were randomly assigned to 6 groups: (1) normal glucose group (5.5 mM); (2) normal glucose + hypoxia-reoxygenation group (H/R); (3) high glucose group (HG) $(30 \mathrm{mM})$; (4) high glucose + hypoxia-reoxygenation group (HH/R); (5) high glucose + hypoxia-reoxygenation + sulforaphane group (HH/R + SFN); (6) high glucose + hypoxia-reoxygenation + sulforaphane + erastin group $(\mathrm{HH} / \mathrm{R}+\mathrm{SFN}+\mathrm{Era}) . \mathrm{H} 9 \mathrm{c} 2$ cardiomyocytes of all groups were cultured in low-glucose DMEM containing $10 \%$ FBS and $1 \%$ penicillin/streptomycin and incubated in 
normoxic incubator at $37{ }^{\circ} \mathrm{C}$ in a humidified atmosphere of $5 \% \mathrm{CO}_{2}$. When the density of $\mathrm{H} 9 \mathrm{c} 2$ cardiomyocytes reached $70-80 \%$, all cells were digested with trypsin containing ethylenediaminetetraacetic acid (EDTA) and transferred to 6-well culture plates for subsequent experimental treatments. To simulate the high glycemic state, cells were cultured in serum-free medium overnight and exposed to HG medium for $24 \mathrm{~h}$. For the H/R cell model, cells were subjected to hypoxic state $\left(94 \% \mathrm{~N}_{2}+5 \% \mathrm{CO}_{2}+1 \% \mathrm{O}_{2}\right)$ for $6 \mathrm{~h}$ and followed by reoxygenation $\left(95 \%\right.$ air $\left.+5 \% \mathrm{CO}_{2}\right)$ for $2 \mathrm{~h}$. Sulforaphane and erastin was given $24 \mathrm{~h}$ before $H / R$. The experimental protocol is depicted schematically in Fig. 1.

\section{Determination of myocardial infarction}

After $2 \mathrm{~h}$ of ischemia-reperfusion treatment, six rats in each group were randomly selected to ligate LAD along with the original site. The $2 \%$ Evans Blue reagent was slowly injected from the femoral vein, while the nonischemic area showed blue staining region. The aorta was immediately clamped and the heart was removed and sliced along the longitudinal axis. Five pieces of heart slices were prepared, placed in the $1 \%$ TTC solution in a $37{ }^{\circ} \mathrm{C}$ incubator for $30 \mathrm{~min}$ in the dark. The slices were then fixed in $4 \%$ paraformaldehyde for 30 min, scanned by the scanner (Epson, v30, Japan). And the myocardium area was calculated with an image analysis system (Image J; National Institutes of Health, USA). Theoretically, normal myocardium appears blue, ischemic myocardium shows brick-red, and infarcted myocardium displays pale. For ischemic myocardium stained by TTC staining, brick-red myocardium was defined as the area at risk (AAR) and pale myocardium was defined as the infarct area (IA). The percentage of myocardial infarction area was calculated as IA versus AAR $($ IA/AAR $\times 100 \%)$.

\section{Echocardiography}

In order to avoid the interference of residual gas in the thoracic cavity on the echocardiography after thoracotomy, 6 rats were taken from each group after $72 \mathrm{~h}$ of reperfusion. Rats were anesthetized with 2-3\% inhaled isoflurane and fixed on the operating table. The MyLab 30CV ultrasound system (Biosound Esaote, Indianapolis, IN, USA) with a $15-\mathrm{MHz}$ transducer probe was used to perform two-dimensional and M-mode echocardiographic measurements. Parasternal long axis and short axis images were obtained in short and long axes in two-dimensional and M-mode for quantification. Left ventricular internal dimension systole (LVIDs) and left ventricular internal dimension diastole (LVIDd) were measured on the parasternal LV long axis view. Left ventricular ejection fraction (LVEF) and left ventricular shortening fraction (LVFS) were calculated by computer algorithms. All measurements that represented the mean of 5 consecutive cardiac cycles were performed in a blinded manner.

\section{Hematoxylin and eosin staining}

Myocardial tissue was fixed overnight in 4\% paraformaldehyde at $4{ }^{\circ} \mathrm{C}$. The tissue was then dehydrated in a series of ethanol and xylene solutions and embedded in paraffin wax. A microtome was used to slice the heart tissue into slices of $4 \mu \mathrm{m}$ thick. Hematoxylin and eosin (HE) staining
Fig. 1 Experimental protocols. Illustration showing the experimental procedures including diabetes induction, ischemiareperfusion model, SFN and Era administration, and sacrifice end point. A In vivo experimental protocol. B In vitro experimental protocol. STZ streptozotocin, $S F N$ sulforaphane, Era erastin
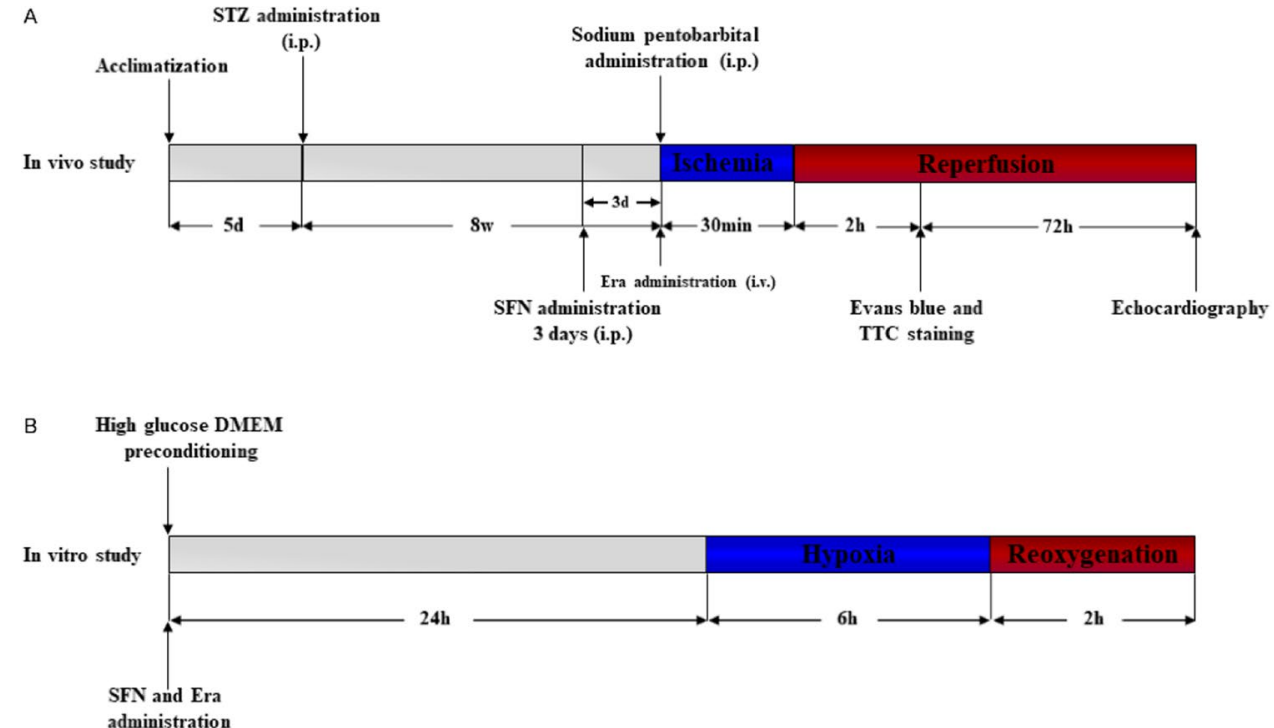
was used to examine the morphological changes and damage severity as instructed by the manufacturer.

\section{Measurement of serum CK-MB and LDH levels}

At the end of reperfusion, arterial blood samples were collected at the apex of the heart and centrifuged at $1200 \mathrm{rpm}$ for $15 \mathrm{~min}$. The creatine kinase-MB (CK-MB) was detected by the assay kit and lactate dehydrogenase (LDH) was detected by colorimetry according to the manufacturer's instructions.

\section{Cell viability assay}

Cells were plated in 96-well plates at a density of 3000 cells/well and grouped according to the experiment (5 replicate wells per group). After cells were cultured and treated in 96 -well plates, $10 \mu \mathrm{L}$ of CCK- 8 reagent was added to each well and then incubated for $2 \mathrm{~h}$ in the dark. Absorbance was detected at $450 \mathrm{~nm}$ with a microplate reader (Perkin Elmer, USA).

\section{$M D A, S O D$, and $\mathrm{Fe}^{2+}$ determination}

After modeling, the cells and cell supernatant were collected to measure the MDA level, antioxidant enzyme SOD activity and intracellular $\mathrm{Fe}^{2+}$ concentration by spectrophotometry according to the manufacturer's instructions. Then, the intensity was observed by a microplate reader.

\section{ROS measurement}

Intracellular reactive oxygen levels were measured with the 2',7'-dichlorofluorescein diacetate (DCFH-DA) molecular probe. Briefly, cardiomyocytes in 6-well plates were loaded with the appropriate amount of DCFH-DA probe for $30 \mathrm{~min}$ at $37^{\circ} \mathrm{C}$ in the dark. DCFH-DA was oxidized and converted to highly fluorescent DCFH and showed green fluorescence in the cytoplasmic lysate. Fluorescence images were captured by a fluorescence microscope (Olympus, Tokyo, Japan).

\section{Immunofluorescence}

H9c2 cardiomyocytes in 6-well plates were fixed with $4 \%$ paraformaldehyde for $30 \mathrm{~min}$. After that, they were permeabilized in $0.5 \%$ Triton $\mathrm{X}-100$ at $37{ }^{\circ} \mathrm{C}$ for 20 min, blocked with 5\% BSA solution and add NRF2 (1:100, ABclonal, Wuhan, Hubei, China) antibody to incubate overnight at $4{ }^{\circ} \mathrm{C}$. Finally, the samples were incubated with $\mathrm{Cy} 3$ labeled goat anti-rabbit secondary antibody, followed by 4',6-diamidino-2-phenylindole dihydrochloride (DAPI) (Invitrogen, Carlsbad, CA, USA) staining for $10 \mathrm{~min}$, then observed and captured positive areas with a laser confocal microscope (Leica TCS, Germany).

\section{Quantitative real-time polymerase chain reaction (qRT-PCR) analysis}

Total RNA was extracted from the myocardial samples and H9c2 cells using Trizol reagent. Two micrograms of RNA from each sample was then reverse transcribed into cDNA according to the Prime-Script RT reagent kit instruction (Servicebio, Wuhan, Hubei, China). The qRT-PCR was performed using a SYBR Green qPCR Reagent Kit (Servicebio, Wuhan, Hubei, China) by Bio-Rad CFX Connect Real-Time PCR Detection System (Bio-Rad, USA). The used primers are listed in Table 1. The mRNA levels were normalized to $\beta$-actin mRNA level. The expression of genes was analyzed by using the $2^{-\Delta \Delta C T}$ method.

\section{Western blotting}

H9c2 cardiomyocytes and cardiac tissue were homogenized in pre-cooled RIPA lysis buffer following various treatments. The supernatant was taken and added to the protein buffer. Then, the extracts were placed at $100{ }^{\circ} \mathrm{C}$ and keep them for $5 \mathrm{~min}$. Fifty micrograms of total protein per well was separated by SDS-PAGE gel and then transferred to PVDF membranes. For $1 \mathrm{~h}$, the membranes were incubated in 5\% skimmed milk. Primary antibodies (ACSL4, 1:1000; GPX4, 1:1000; NRF2, 1:1000; FPN1, 1:1000; GAPDH, $1: 1000)$ were incubated with the membranes overnight at $4{ }^{\circ} \mathrm{C}$. Secondary antibody (1:5000, Proteintech) was incubated for $1 \mathrm{~h}$ at room temperature with horseradish
Table 1 The sequences of RT-PCR used in this study

\begin{tabular}{lll}
\hline Gene & Forward $\left(5^{\prime}-3^{\prime}\right)$ & Reverse $\left(5^{\prime}-3^{\prime}\right)$ \\
\hline ACSL4 & CTGCCGAGTGAATAACTTTGGA & TCAGATAGGAAGCCTCAGACTCATT \\
NRF2 & TTGGGGTAAGTCGAGAAGTGTTT & ATGTGGGCAACCTGGGAGTA \\
SLC40A1 & CTAAATCCGTCCCCATAATCTCC & CCCATTGCCACAAAGGAGAC \\
$\beta$-actin & TGCTATGTTGCCCTAGACTTCG & GTTGGCATAGAGGTCTTTACGG \\
\hline
\end{tabular}


peroxidase (HRP) conjugated secondary antibody. The biological image analysis system was used for the final analysis (Bio-Rad, USA). Each sample's GAPDH levels were used to determine the relative expression levels of the target proteins.

\section{Statistical analysis}

All results were analyzed using Graphpad Prism 8.0 software (GraphPad Software, USA) and presented as means \pm standard deviation. Statistical analysis was carried out using one-way analysis of variance (ANOVA) and $P<0.05$ was deemed significant.

\section{Results}

\section{The exacerbated myocardial IRI in diabetic rats was associated with the activation of ferroptosis and the downregulation of the NRF2/FPN1 pathway}

After STZ-induced diabetes, the diabetic rats showed characteristic symptoms of diabetes including hyperglycemia, polydipsia, polyphagia, and weight reduction (Fig. 2A). Moreover, STZ-induced rats exhibited markedly impaired IPGTT and OGTT (Fig. 2B). Therefore, these data demonstrated that our diabetic animal models were successfully developed.

Ischemia-reperfusion significantly impaired cardiac function by decreasing LVEF and LVFS in both diabetic and non-diabetic animals. However, compared with the NIR group, DIR group showed further impaired cardiac function (Fig. 2C). Uniformly, compared with the NIR group, the diabetes markedly increased myocardial infarction area after ischemia-reperfusion treatment (Fig. 2D). In addition, compared with normal rats, diabetic rats had higher levels of LDH and CK-MB (Fig. 2E). HE staining showed that the structure of myocardial tissue in NS group was intact and the cardiomyocytes were arranged neatly. The myocardial tissue in DS and NIR groups showed mild disorder of cell arrangement and partial myocardial borders breakage. In the DIR group, the myocardial tissue showed severe cell alignment disorder, cell swelling, and necrosis, and most of the myocardial fibers were tremendously broken (Fig. 2F). The above results indicated that the myocardial IRI was significantly exacerbated in diabetic rats compared with non-diabetic rats.

Next, to determine the level of transcription in cardiomyocytes NRF2 and FPN1, we assessed the mRNA in cardiomyocytes in each group. Our experiments found that compared with the NS group, the protein and mRNA expression of NRF2 and FPN1 in DS and NIR group was lower (Fig. 2G-H). The mRNA and protein expression levels of NRF2 and FPN1 were significantly reduced in the DIR group. Western blot showed that compared with the NS group, the expression of ACSL4 in the DS group and the NIR group increased, and the expression of ACSL4 in the DIR group further increased (Fig. 2G). However, the level of GPX4 was opposite to ACSL4. And the expression level of mRNA of ACSL4 is consistent with protein (Fig. $2 \mathrm{H}$ ). These data indicated that following myocardial ischemia-reperfusion treatment, impairment of the NRF2/FPN1 signaling pathway caused development of ferroptosis to exacerbate the myocardial IRI, in diabetic rats compared with that in non-diabetic rats.

\section{The ferroptosis inducer abrogated the protective effect of NRF2 activator SFN in diabetic rats following myocardial IRI}

To verify the contribution of NRF2/FPN1-dependent ferroptosis to diabetic myocardial IRI, the NRF2 activator SFN was applied to activate the NRF2/FPN1 signaling pathway. We found that activation of NRF2 could distinctly alleviate myocardial IRI in diabetic rats. SFN significantly improved post-myocardial IRI cardiac functional recovery by increasing LVEF and LVFS, and the size of myocardial infarction was also reduced $(P<0.05)$. While the ferroptosis inducer Erastin attenuated cardioprotective effect of SFN (Fig. 3A and B), the LDH and CK-MB levels in the DIR + SFN group were lower compared with the DIR group (Fig. 3C), indicating that the degree of myocardial IRI was reduced after SFN administration. Through HE staining observation, compared with the DIR group, the left ventricular structural damage in the DIR + SFN group were reduced (Fig. 3D).

Western blot and qRT-PCR showed that after pretreatment with SFN, the expression of NRF2, FPN1, and GPX4 in rat myocardium were upregulated, the expression of ACSL4 was downregulated (Fig. 3E and F). When exposed to Erastin treatment, the above effects of SFN were all inhibited, and the expression of GPX4 was lower than that in DIR + SFN group, but the expression of ACSL4 was higher (Fig. 3A-F). These results collectively suggest that the protective effect on myocardial IRI by activating the NRF2/ FPN1 signaling pathway was achieved through inhibition of ferroptosis.

\section{Downregulation of NRF2/FPN1 pathway was accompanied by ferroptosis in $\mathrm{H} 9 \mathrm{c} 2$ cardiomyocytes exposed to $\mathrm{HH} / \mathrm{R}$}

In order to identify further molecular mechanism of NRF2/ FPN1 pathway in diabetic myocardial IRI, H9c2 cardiomyocytes were used for in vitro experiments. Compared 
A

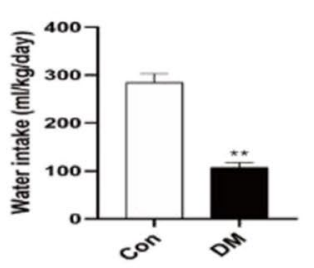

B

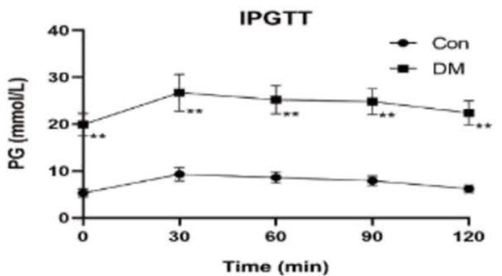

C
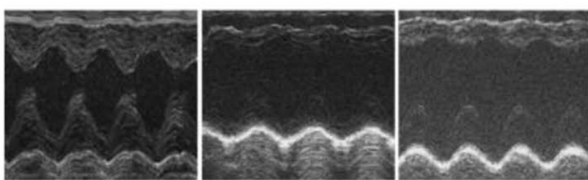

NS

D

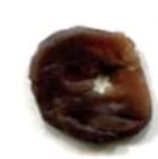

NIR

F
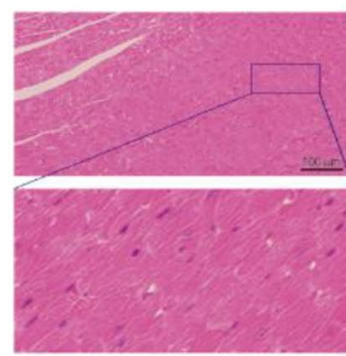

NS

G

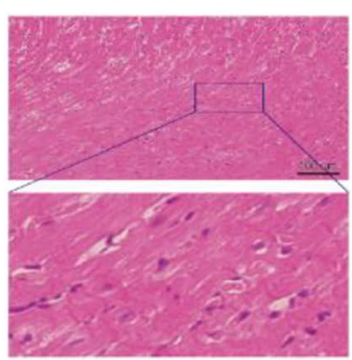

NIR

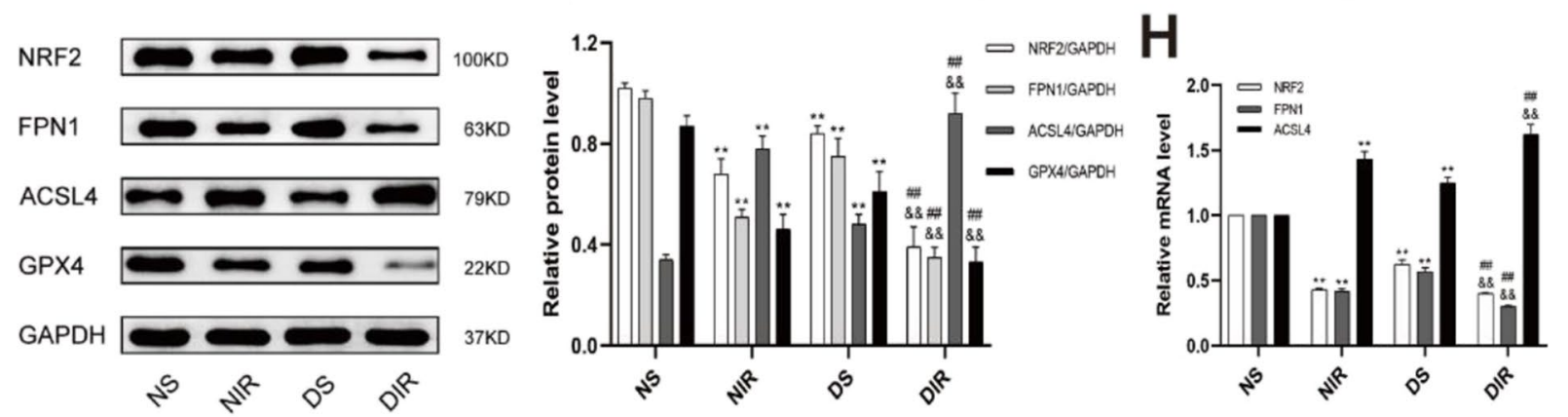

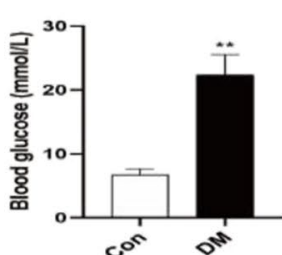
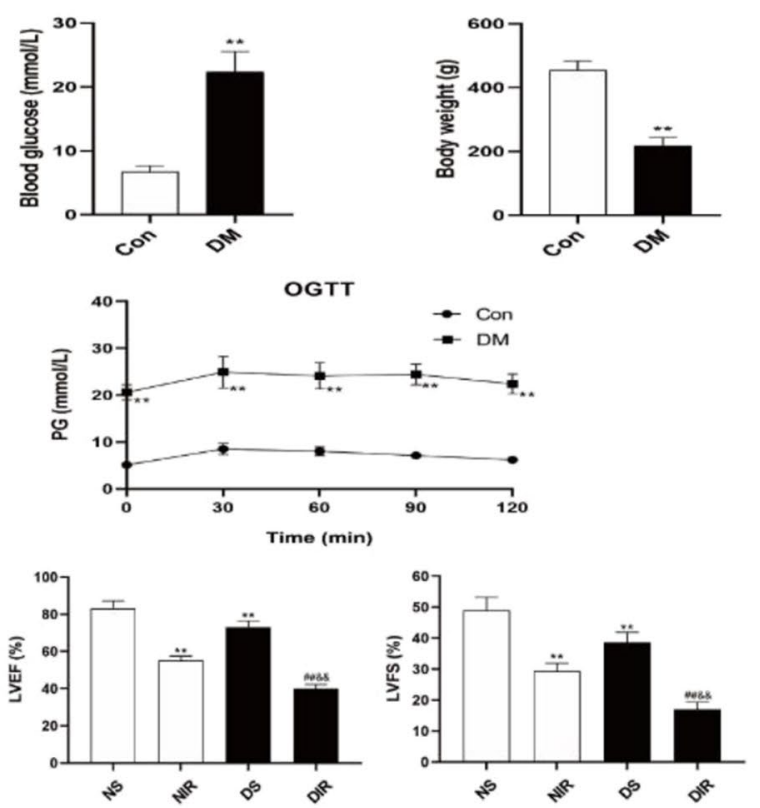

E
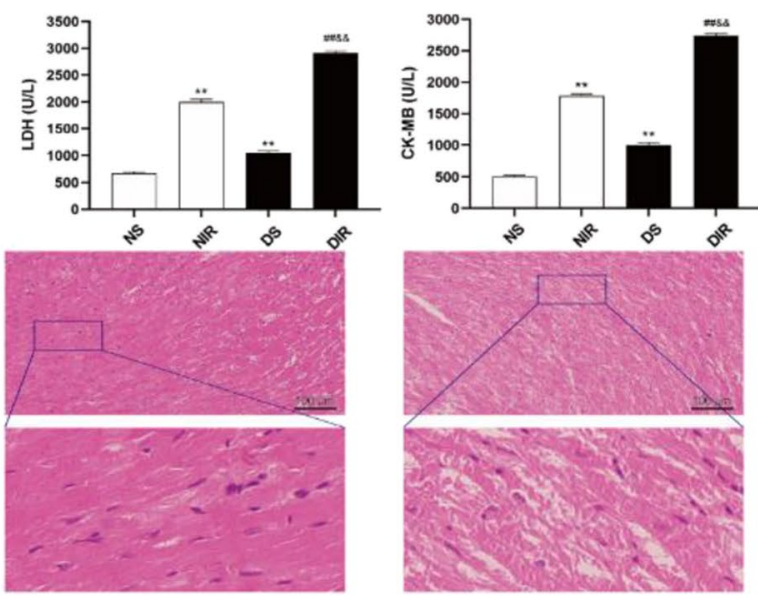

DS

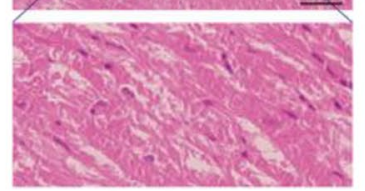

DIR
Fig. 2 The effects of diabetes and myocardial IRI on ferroptosis and NRF2 pathway. A Basic characteristics of diabetic rats. Water intake and food consumption were the average value of 8 weeks. Body weight and plasma glucose were recorded before inducing myocardial ischemia-reperfusion. B Intraperitoneal glucose tolerance test and oral glucose tolerance test of diabetic rats. C Representative M-mode images by echocardiography. D Infarct size was detected by TTC. E The levels of CK-MB and LDH in serum were detected. F Histo- pathological changes of myocardium were detected by HE staining. G The protein levels of NRF2, FPN1, ACSL4, and GPX4 in myocardial tissue were detected by western blot. $\mathbf{H}$ The mRNA levels of NRF2, ACSL4, and GPX4 in myocardial tissue were detected by qRT-PCR. The results are expressed as the means \pm SD. $N=8$ for each group. ${ }^{* *} P<0.05$ vs. NS group. ${ }^{\# \#} P<0.05$ vs. NIR group. ${ }^{\& \&} P<0.05$ vs. DS group. IPGTT intraperitoneal glucose tolerance test, $O G T T$ oral glucose tolerance test 


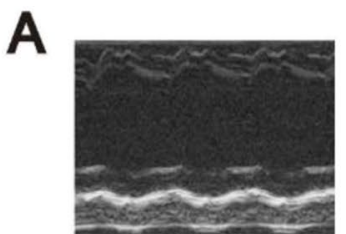

DIR

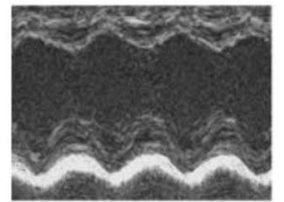

$\mathrm{DIR}+\mathrm{SFN}$

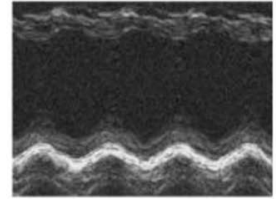

$\mathrm{DIR}+\mathrm{SFN}+\mathrm{Era}$
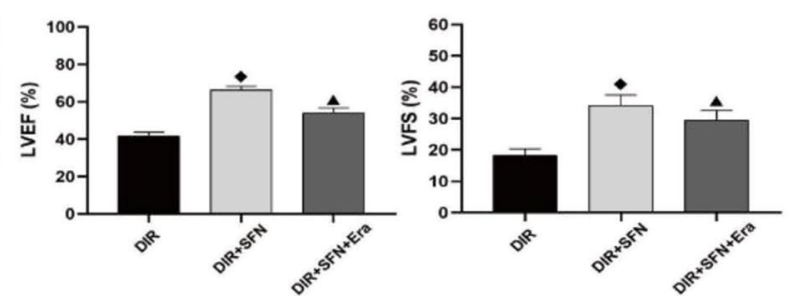

C
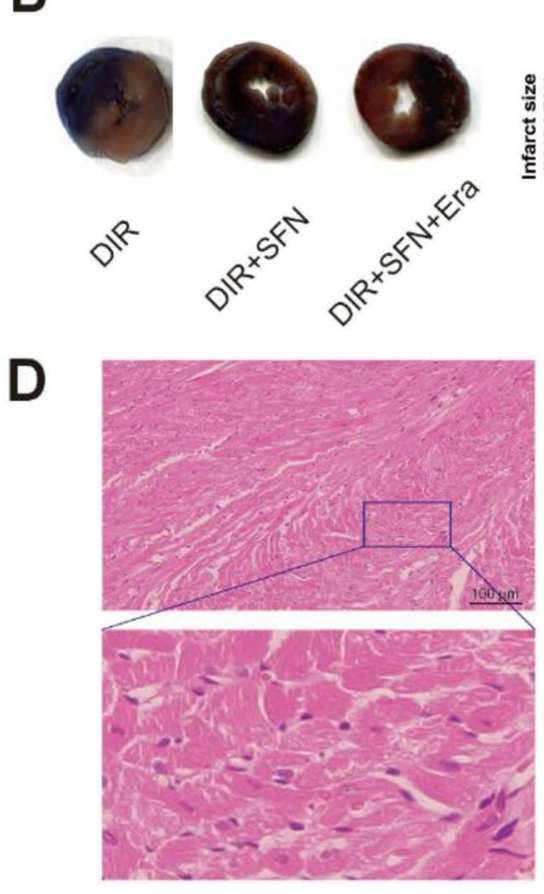

DIR

E
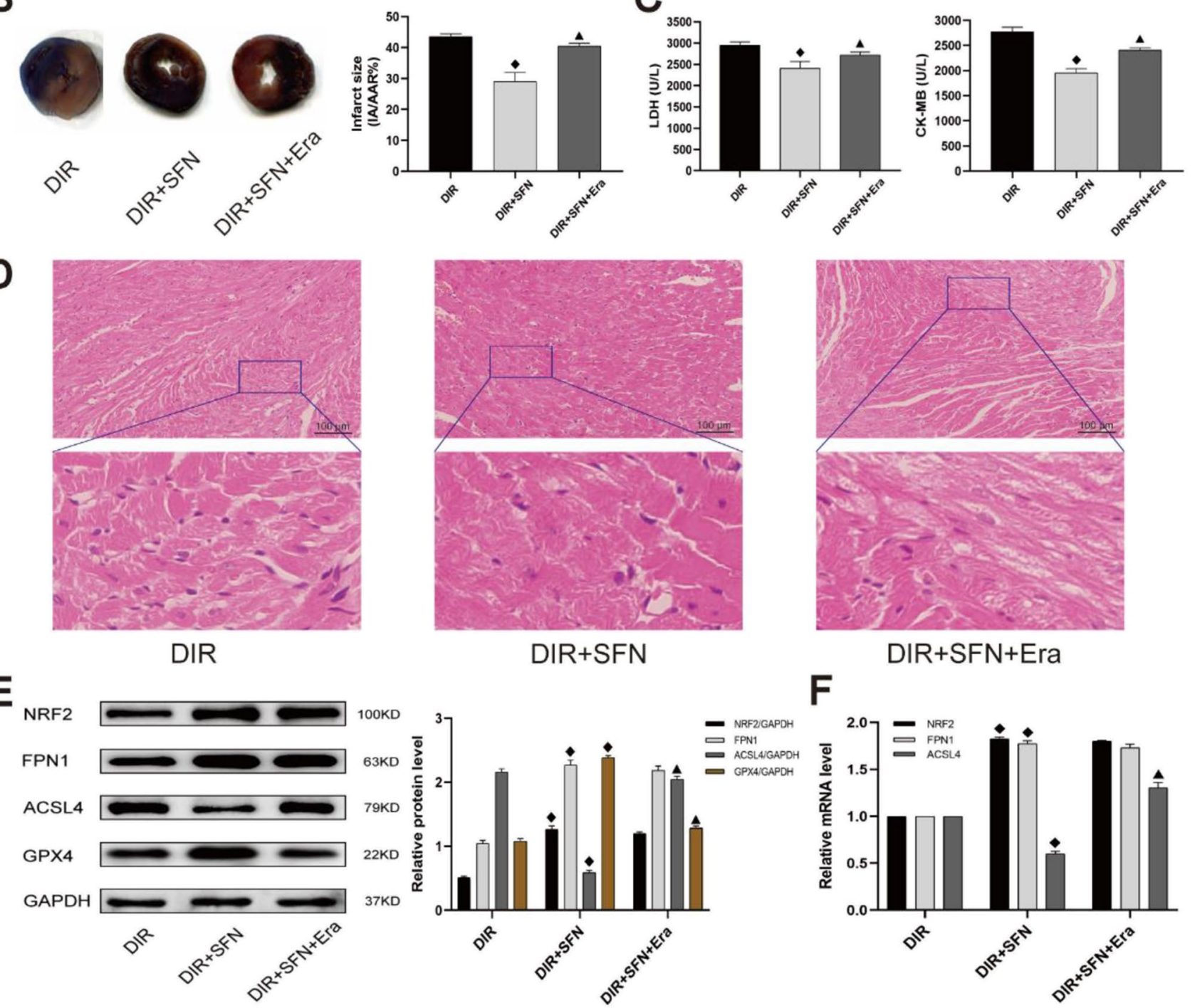

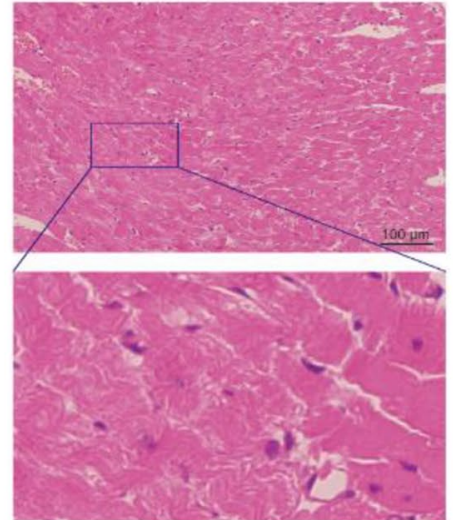

DIR+SFN
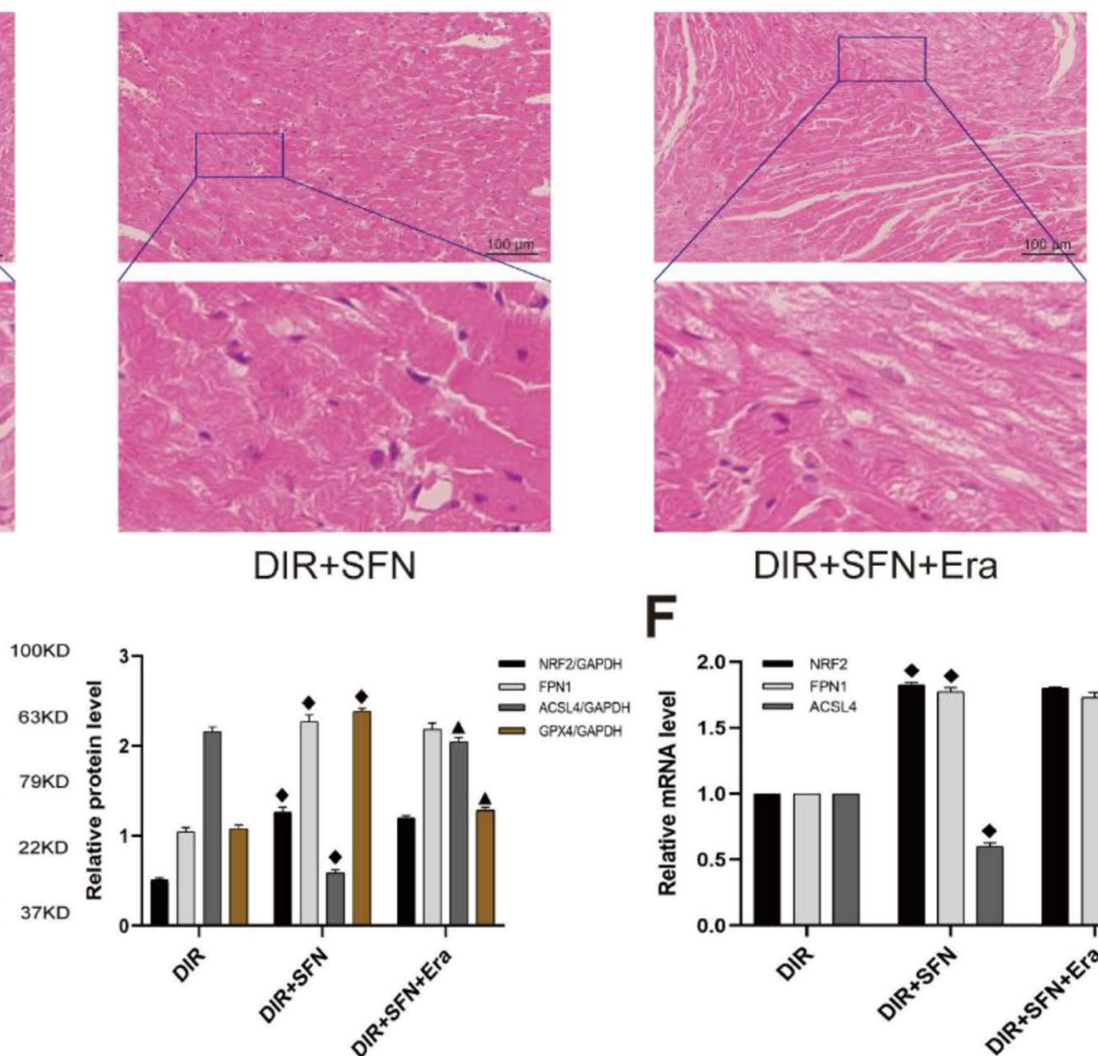

$\mathrm{DIR}+\mathrm{SFN}+\mathrm{Era}$
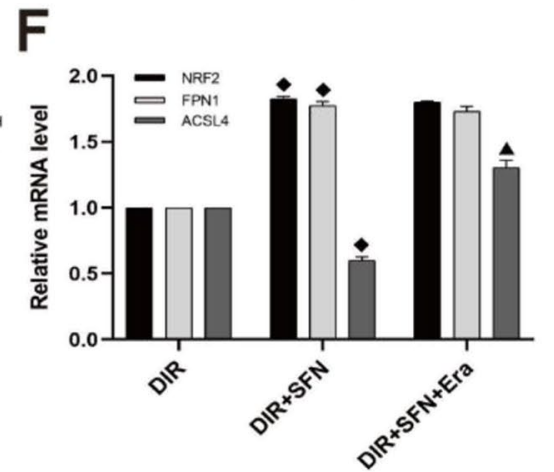

Fig. 3 The ferroptosis inducer could abrogate the protective effect of activating NRF2 pathway in diabetic rats following myocardial IRI. A Representative M-mode images by echocardiography. B Infarct size was detected by TTC. C The levels of CK-MB and LDH in serum were detected. D Histopathological changes of myocardium were detected by HE staining. E The protein levels of NRF2, FPN1,

with normal group, the cell viability of $\mathrm{H} 9 \mathrm{c} 2$ cells in HG group and H/R group were decreased, and lipid ROS production were increased (Fig. 4A and B). HG and H/R
ACSL4, and GPX4 in myocardial tissue were detected by western blot. F The mRNA levels of NRF2, ACSL4, and GPX4 in myocardial tissue were detected by qRT-PCR. The results are expressed as the means $\pm \mathrm{SD} . N=8$ for each group. $P<0.05$ vs. DIR group. ${ }^{\boldsymbol{\Delta}} P<0.05$ vs. DIR + SFN group. $S F N$ sulforaphane, Era erastin

treatment increased intracellular $\mathrm{Fe}^{2+}$ and MDA content and decrease SOD activity (Fig. 4C). And the above changes were more obvious in the HH/R group $(P<0.05)$. 


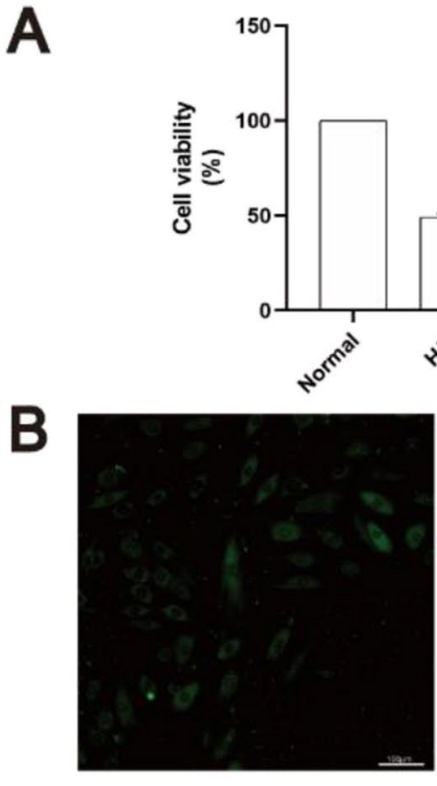

Normal

\section{C}

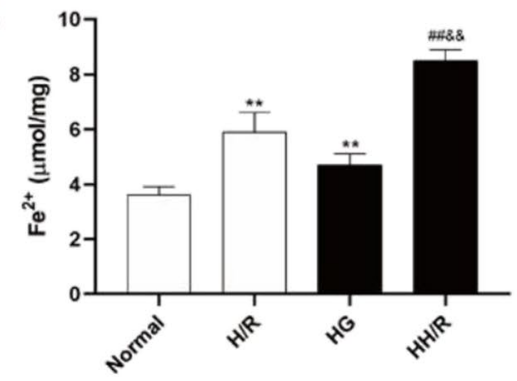

D

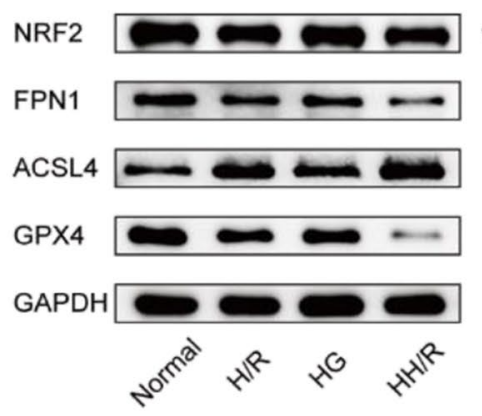

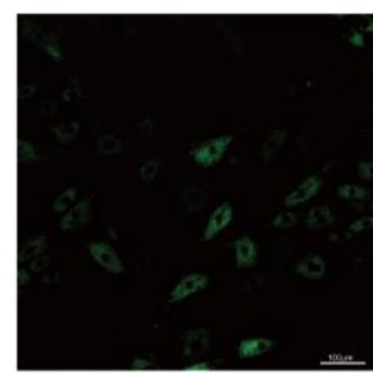

$H / R$
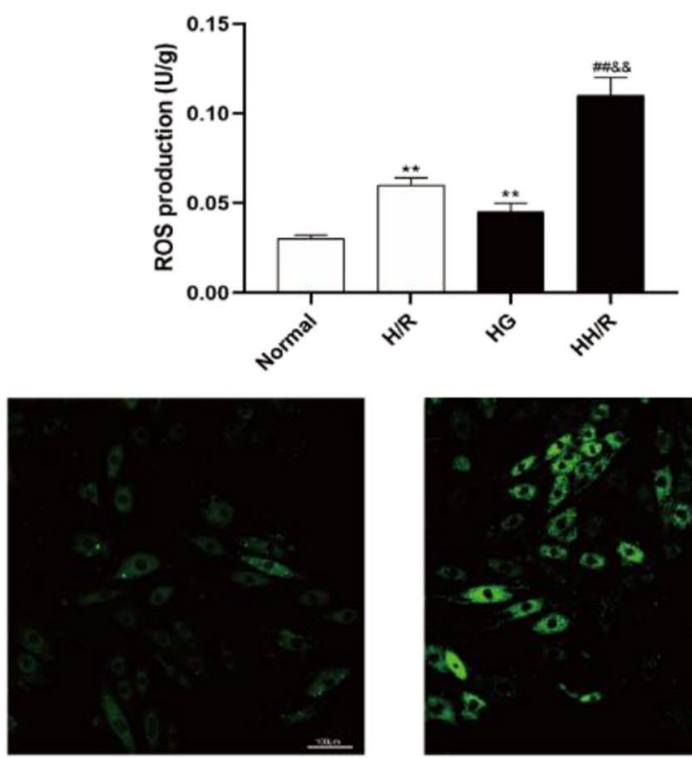

HG

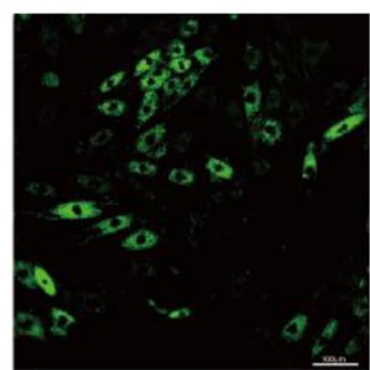

$\mathrm{HH} / \mathrm{R}$
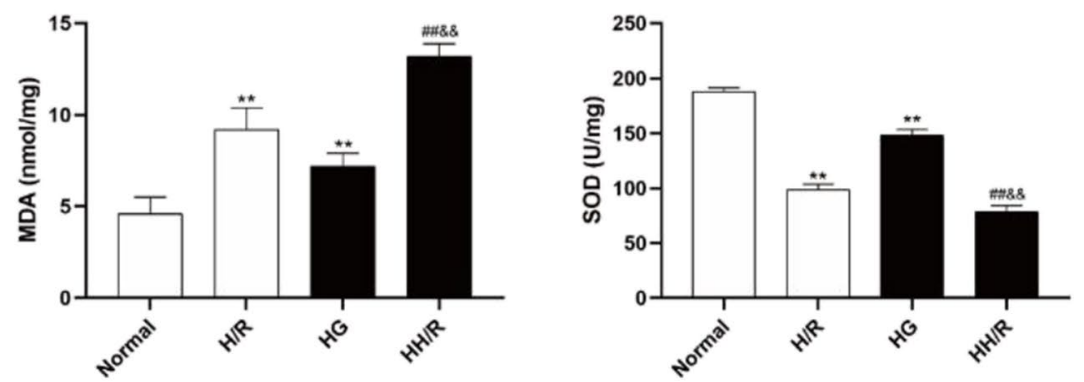

E
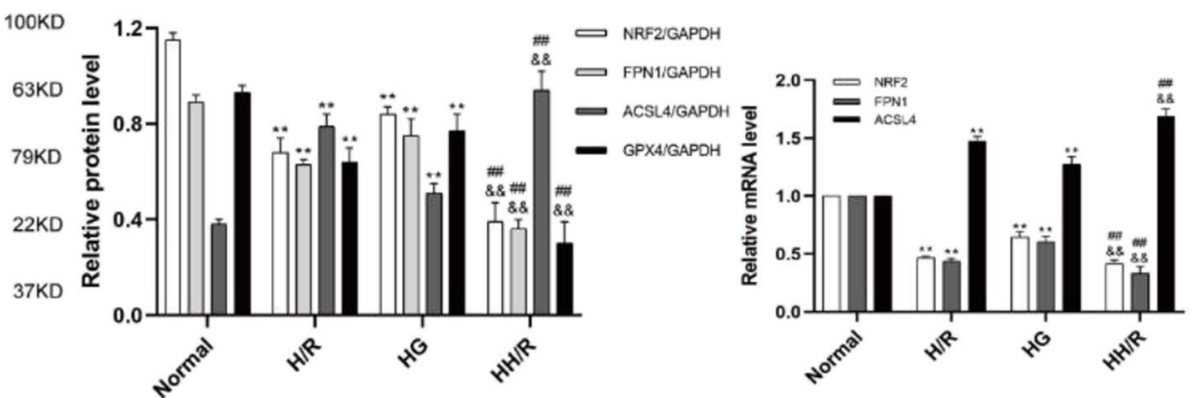

Fig. 4 Downregulation of NRF2 pathway is accompanied by ferroptosis in $\mathrm{H} 9 \mathrm{c} 2$ cardiomyocytes exposed to $\mathrm{HH} / \mathrm{R}$. A Cell viability was detected by CCK-8. B ROS production was measured by adding the fluorescent probes to the cells during $20 \mathrm{~min}$ at $37^{\circ} \mathrm{C}$. Scale bars: $100 \mu \mathrm{m} . \mathrm{C} \mathrm{Fe}^{2+}$ content, MDA, and SOD levels in H9c2 cells were detected. D The protein levels of NRF2, FPN1, ACSL4, and GPX4 in myocardial tissue were detected by western blot. E The mRNA levels of NRF2, ACSL4, and GPX4 in myocardial tissue were detected by qRT-PCR. ${ }^{* *} P<0.05$ vs. NS group. ${ }^{\# \#} P<0.05$ vs. NIR group. ${ }^{\& \&} P<0.05$ vs. DS group
The conditions were consistent with in vitro experiments, and $\mathrm{HG}$ and $\mathrm{H} / \mathrm{R}$ treatment downregulated the expression of NRF2, FPN1, and GPX4 at the same time upregulated the expression of ACSL4 (Fig. 4D and E). From these findings, we could infer that hypo-expression of the NRF2/ FPN1 signaling pathway in $\mathrm{H} 9 \mathrm{c} 2$ cardiomyocytes exposed to $\mathrm{HH} / \mathrm{R}$ could cause iron over-load and lipid peroxide accumulation, which in turn results in ferroptosis. 
The protective effects of NRF2 activator SFN in H9c2 cardiomyocytes from $\mathrm{HH} / \mathrm{R}$-induced ferroptosis were reversed by erastin

To identify the possible mechanisms underlying the protective effects of NRF2 in HH/R-induced ferroptosis, H9c2 cardiomyocytes overexpressing NRF2 were obtained via administration with SFN. As depicted in Fig. 5, compared to the other groups, the HH/R and SFN treatment group showed more significant upregulation of NRF2/FPN1 signaling pathway and more pronounced changes in ferroptosis-related proteins $(P<0.05)$. Therefore, in line with our expectations, we focused our study mainly on the HH/R group. As showed in Fig. 6A, the cell viability was increased in the HH/R + SFN group. Compared with HH/R group, the ROS fluorescence intensity was significantly reduced after SFN incubation (Fig. 6B). Besides, SFN treatment significantly attenuated intracellular $\mathrm{Fe}^{2+}$ content and lipid peroxide accumulation by impacting SOD and MDA levels (Fig. 6C). After NRF2 was activated by SFN, the expression of iron metabolism-related protein FPN1 was also increased accordingly, with reduced indicators of ferroptosis (Fig. 6D and E). Furthermore, as seen in Fig. 6A-E, after pretreatment with erastin, the expression of NRF2 and FPN1 did not change significantly with increased ferroptosis level $(P>$ $0.05)$. Collectively, ferroptosis inducer reversed the beneficial effect of overexpression of NRF2/FPN1 signaling pathway to protect H9c2 cardiomyocytes from HH/Rinduced ferroptosis.

\section{NRF2 activator SFN protected H9c2 cardiomyocytes from $\mathrm{HH} / \mathrm{R}$-induced ferroptosis by transcribing downstream iron metabolism-related gene via nuclear translocation}

To visualize the nuclear localization of NRF2, an immunofluorescence assay was performed in $\mathrm{H} 9 \mathrm{c} 2$ cardiomyocytes. As presented in Fig. 7, compared with normal group, there was a significant decrease in NRF2 protein expression in the nucleus relative to the cytoplasm following the treatment of HH/R $(P<0.05)$. Moreover, SFN pretreatment significantly increased the nuclear translocation of NRF2 $(P<0.05)$. Taken together, these data suggested that SFN protected H9c2 cardiomyocytes from ferroptosis damage by modulating NRF2 nuclear accumulation.

\section{Discussion}

In this study, we attempted to understand whether ferroptosis, an iron-dependent regulated form of cell death caused by iron dysmetabolism and accumulation of lipid peroxides, may underlie the pathogenesis of myocardial IRI in diabetic rats. Furthermore, we first evaluated whether the activation of NRF2/FPN1 signal pathway could prevent ferroptosis
Fig. 5 The effects of NRF2 activator SFN on $\mathrm{HG}$ and $\mathrm{H} / \mathrm{R}$ treated $\mathrm{H} 9 \mathrm{c} 2$ cardiomyocytes. ${ }^{*} P<0.05$ vs. vehicle group. ${ }^{\#} P<0.05$ vs. H/R group.

${ }^{\&} P<0.05$ vs. HG group.

$\Delta P<0.05$ vs. HH/R group. $S F N$ sulforaphane
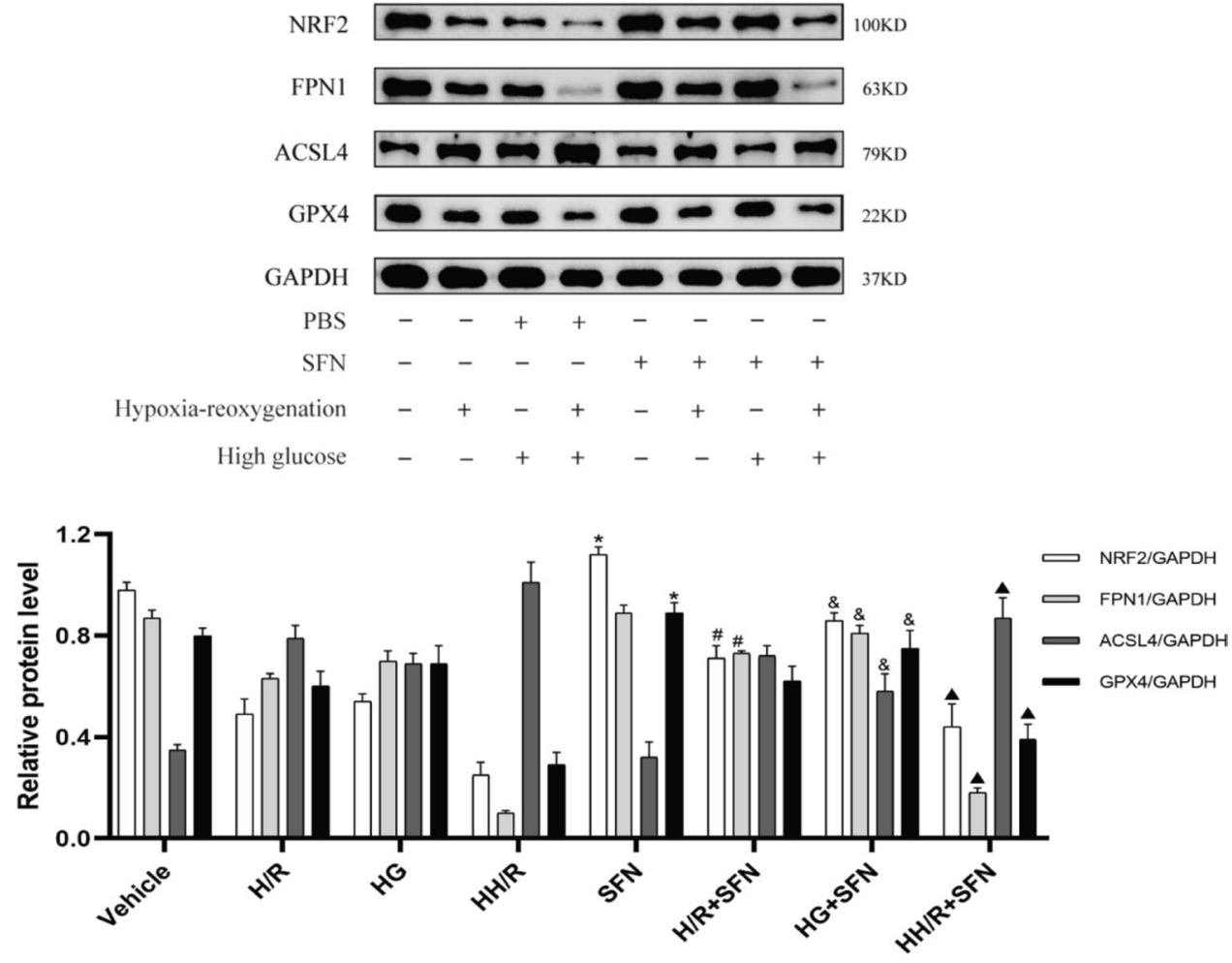
A
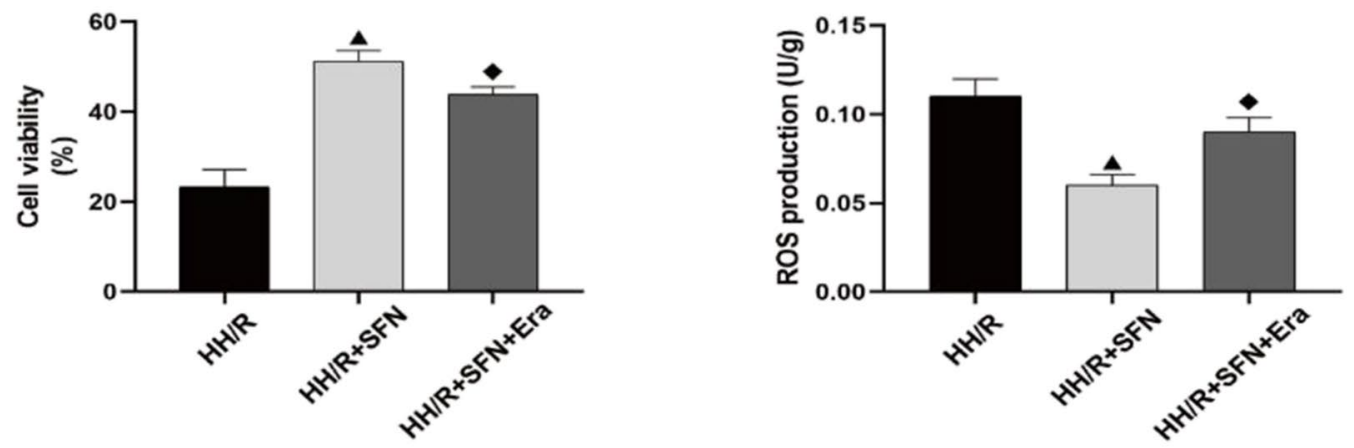
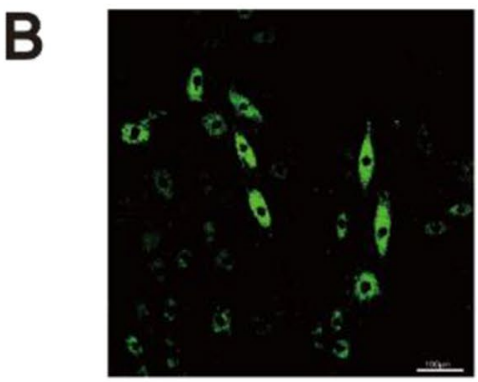

$\mathrm{HH} / \mathrm{R}$

C

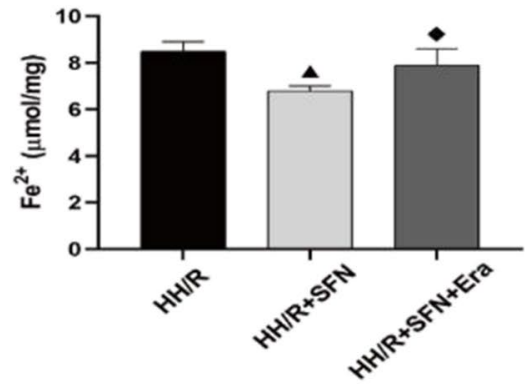

D

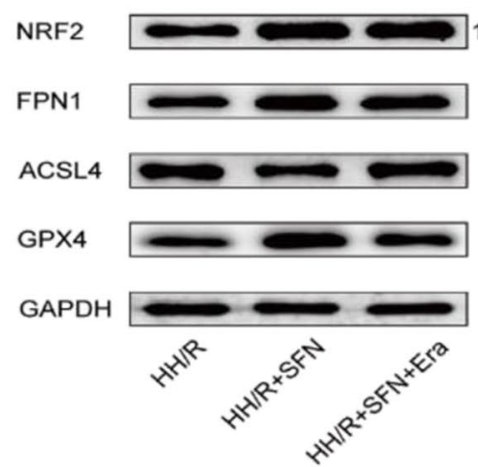

$100 \mathrm{KD}$

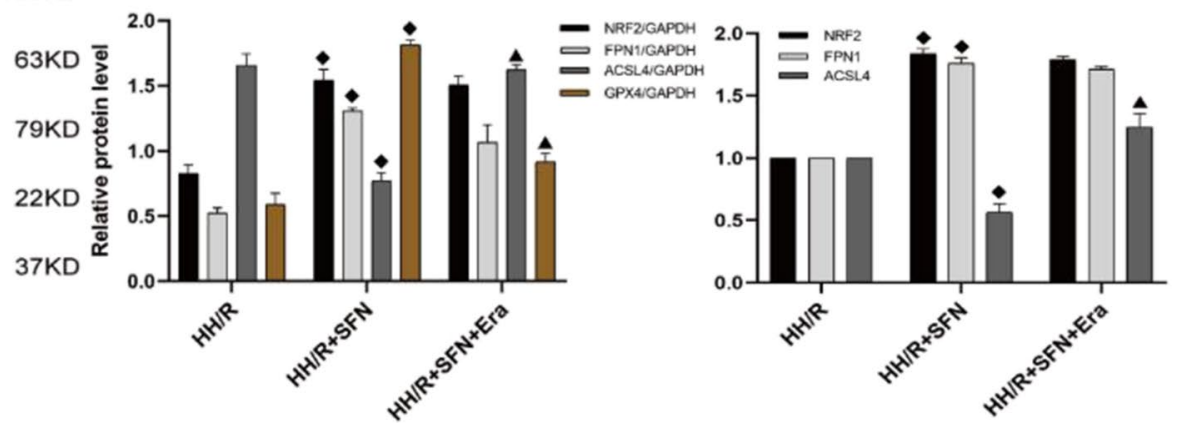

Fig. 6 The beneficial effects of overexpression of NRF2 pathway to protect $\mathrm{H} 9 \mathrm{c} 2$ cardiomyocytes from $\mathrm{HH} / \mathrm{R}$-induced ferroptosis were reversed by erastin. A Cell viability was detected by CCK-8. B ROS production was measured by adding the fluorescent probes to the cells during $20 \mathrm{~min}$ at $37{ }^{\circ} \mathrm{C}$. Scale bars: $100 \mu \mathrm{m}$. $\mathrm{C} \mathrm{Fe}^{2+}$ content, MDA, and SOD levels in $\mathrm{H} 9 \mathrm{c} 2$ cells were detected. D The protein levels of
NRF2, FPN1, ACSL4, and GPX4 in myocardial tissue were detected by western blot. E The mRNA levels of NRF2, ACSL4, and GPX4 in myocardial tissue were detected by qRT-PCR. $P<0.05$ vs. DIR group. ${ }^{\boldsymbol{}} P<0.05$ vs. DIR $+\mathrm{SFN}$ group. $S F N$ sulforaphane, Era erastin 

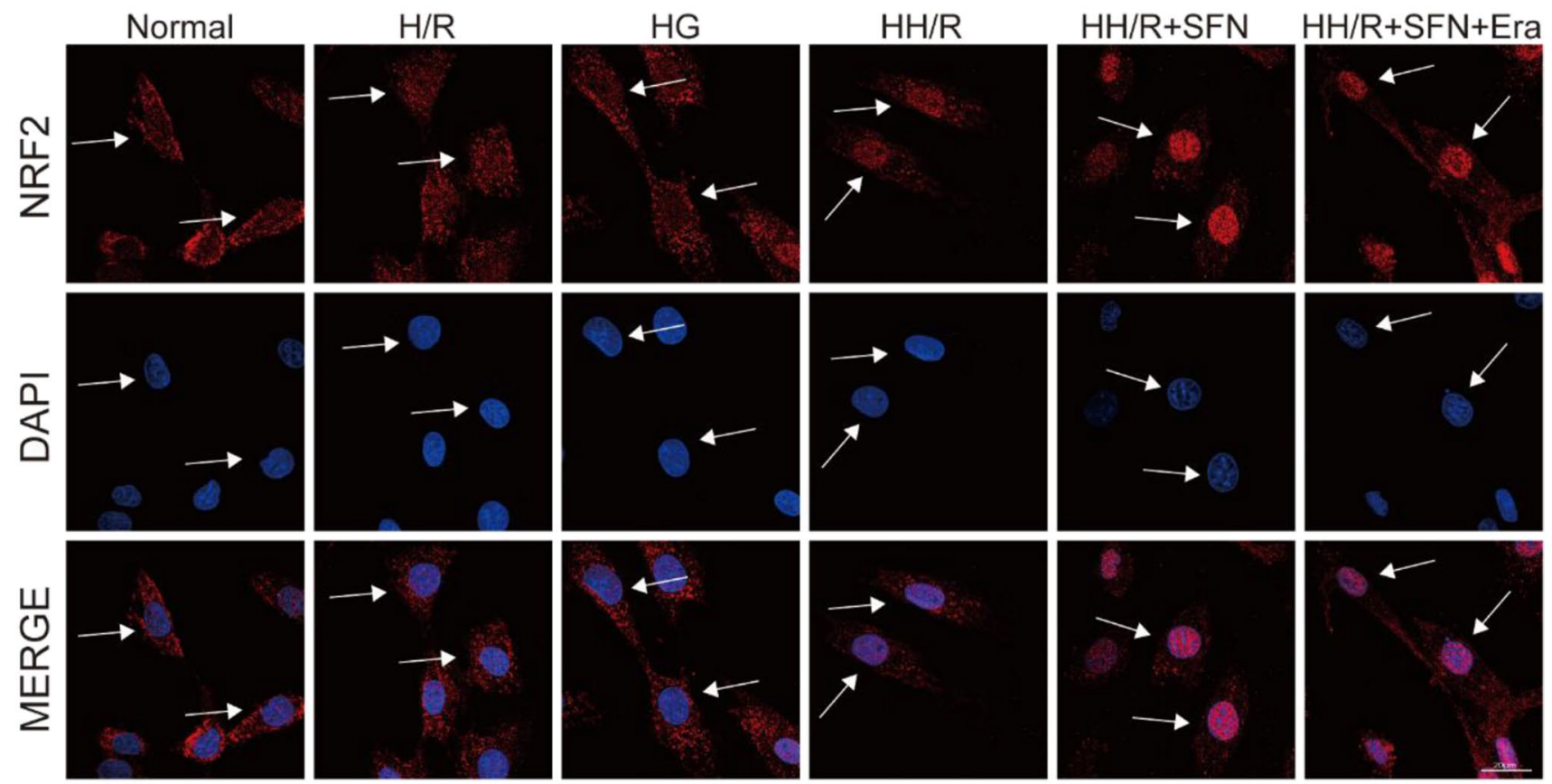

Fig. 7 Immunofluorescence staining of NRF2 using H9c2 cardiomyocytes after corresponding stimulation. The red dots represent NRF2, and blue dots show nuclear. Scale bars: $20 \mu \mathrm{m}$. SFN sulforaphane, Era erastin

in diabetic myocardial IRI. In addition, nuclear transfer of NRF2 plays a pivotal role in ferroptosis in STZ-induced diabetic IRI. To our knowledge, this is the first study to examine the mechanism of NRF2-mediated cardio-protection by regulating iron metabolic balance and iron homeostasis.

In the present study, we respectively established diabetes and ischemia-reperfusion models in rats to simulate myocardial ischemia-reperfusion in diabetic patients. For the in vivo experiments, it was obviously showed that diabetes could increase the vulnerability of the heart following ischemia-reperfusion. When the body's tissues or organs are damaged, cellular metabolism gets affected and $\mathrm{PH}$ decreases, resulting in the reduction of intracellular $\mathrm{Fe}^{3+}$ to $\mathrm{Fe}^{2+}$, overloaded iron ions can form highly reactive and toxic hydroxyl radicals via the Haber-Weiss/Fenton reactions, which in turn can cause ferroptosis (Qi et al. 2020). Furthermore, related research has shown that supply of exogenous iron such as ferric ammonium citrate, ammonium citrate, and ferric citrate can enhance the ferroptosis induced by erastin and RSL3 (Manz et al. 2016), while the antioxidant vitamin $\mathrm{E}$ can inhibit ferroptosis (Kagan et al. 2017). It is thus clear that iron metabolic homeostasis and oxidative stress play key roles in the pathogenesis of ferroptosis. The final product of membrane lipid peroxidation reaction, MDA, might reflect the degree of lipid peroxidation reaction beginning. SOD, which is commonly found in aerobic organisms, is an important member of the antioxidant enzyme class and is one of the main enzymes for effective scavenging of ROS in organisms. By measuring the content of $\mathrm{Fe}^{2+}$, SOD, and MDA in myocardial tissues, our study found iron ions accumulation and imbalance of oxidative and antioxidant systems in rats' hearts under diabetes and myocardial IR conditions. We therefore hypothesized that ferroptosis may have occurred during diabetic myocardial IRI. Acyl-CoA synthetase long-chain family member 4 (ACSL4) is a reliable marker of ferroptosis and induces the production of the signature signal of ferroptosis, 5-hydroxyeicosatetraenoic acid (5-HETE) (Yuan et al. 2016). GPX4 is the only enzyme in the GPX family that reduces esterification of oxidized fatty acids and cholesterol hydroperoxides (Doll et al. 2019). As we expected, the mRNA and protein expression of ACSL4 and GPX4 were changed during diabetic myocardial IRI, which was in line with previous reports (Wang et al. 2020; Zhao et al. 2017). Notably, our previous study also confirmed the fact that ferroptosis is involved in regulating myocardial IRI in diabetic rats, and these were consistent with our present results ( $\mathrm{Li}$ et al. 2020, 2021). From these, we conclude that the increased vulnerability of myocardial IRI in diabetic patients is strongly linked to the disorders of iron metabolism and ferroptosis.

A growing number of experimental studies have illustrated that NRF2 can attenuate diabetic myocardial IRI and effectuate cardioprotective effects (Zhou et al. 2021). In present study, our results showed that the level of NRF2 was downregulated after IR induction as well as during hyperglycemic states, which was consistent with the conclusion of Liu et al. (Liu et al. 2020b) 
and Tan et al. (Tan et al. 2011). Therefore, we inferred that the downregulation of NRF2 expression in the diabetic heart exacerbates oxidative stress and insulin resistance, resulting in exacerbation of myocardial damage. SFN is a well-studied classic NRF2 activator that prevents oxidative stress injury and the accompanying cardiovascular disease. In consideration of the fact that the pathogenesis of diabetic cardiomyopathy is a complex chronic process, fast drug administration of SFN has limited cardioprotection in diabetic rats. Consequently, we referred to Piao et al. who chose to inject SFN ( $500 \mu \mathrm{g} / \mathrm{kg} / \mathrm{day})$ intraperitoneally for 3 consecutive days before ischemia (Piao et al. 2010). SFN administration dramatically reduced diabetic myocardial IRI, as shown by smaller infarcts, lower serum biomarker levels, and preserved cardiac function, according to our findings. The NRF2 has been widely reported to play a novel role in diabetic myocardial IRI and also participates in ferroptosis (Fang et al. 2019; Xiao et al. 2019). Meanwhile, some studies proved that at the transcriptional level, NRF2 could regulate the expression of FPN1, a protein that exerts an important role in iron homeostasis at the systemic level and is currently the only known iron release related protein in mammals (Donovan et al. 2005; Marro et al. 2010). Additionally, our data suggested that compared with the DIR group, NRF2, FPN1, and GPX4 expressions were upregulated, ACSL4 expression was downregulated, myocardial $\mathrm{Fe}^{2+}$ and MDA content was decreased, and SOD activity was increased in the DIR + SFN group, implying that SFN can reduce myocardial IRI in diabetic rats by promoting FPN1-mediated $\mathrm{Fe}^{2+}$ efflux and in turn limiting ferroptosis.

To further test our hypothesis, the specific mechanism was verified in H9c2 cells in detail. There have been a lot of studies showed the involvement of oxidative stress in diabetes as well as in myocardial IRI (Zhao et al. 2017). ROS has also been shown to cause cell necrosis and tissue damage, either directly or indirectly (Ma 2010). H9c2 cells induced by HG or $\mathrm{H} / \mathrm{R}$ were subjected to severe damage, and their ROS levels were elevated, which was consistent with in vivo experimental data. However, the mRNA and protein expression of NRF2 and FPN1 were decreased. This was consistent with the findings of Xu et al. (Xu et al. 2019). We consider that it may be due to the fact that diabetic heart disease is a chronic disease model where long-term oxidative stress depletes antioxidant elements such as NRF2. In further study, the application of SFN rescued this situation and thereby alleviated HH/R damage in $\mathrm{H} 9 \mathrm{c} 2$ cells. Notably, it has been recently shown that changing NRF2 expression in the nucleus can impact its transcriptional target FPN1, resulting in decreased iron efflux and increased intracellular iron content (Yang et al. 2019). Similarly, in our study, our cellular immunofluorescent analysis revealed that NRF2 translocates from the cytoplasm to the nucleus in the process of $\mathrm{H} 9 \mathrm{c} 2$ cells damage induced by HG or $\mathrm{H} / \mathrm{R}$, thereby activating the transcription of its target gene FPN1 to limit ferroptosis. Overall, in response to oxidative stress, we speculated that NRF2 was released from the cytoplasm and translocated into the nucleus, where it bound to the upstream promoter region of the antioxidant response element (ARE) and members of the sMaf protein family. As shown in Fig. 8, the above process eventually resulted in transcription of downstream protective protein genes like FPN1 and heightened cellular resistance to ferroptosis.

Erastin, a ferroptosis inducer that acts on the cystine/ glutamate antiporter system Xc-, is the most widely
Fig. 8 The effects of NRF2 activation on iron metabolism in cardiomyocytes. After SFN pretreatment, activated NRF2 translocates from the cytoplasm to the nucleus. Subsequently, NRF2 forming a heterodimer with small MAFs (SM) enhances the transcription of FPN1. Overloaded free iron is exported to the plasma via FPN1, which proves the protective effect of the NRF2 induction against ferroptosis. SFN sulforaphane

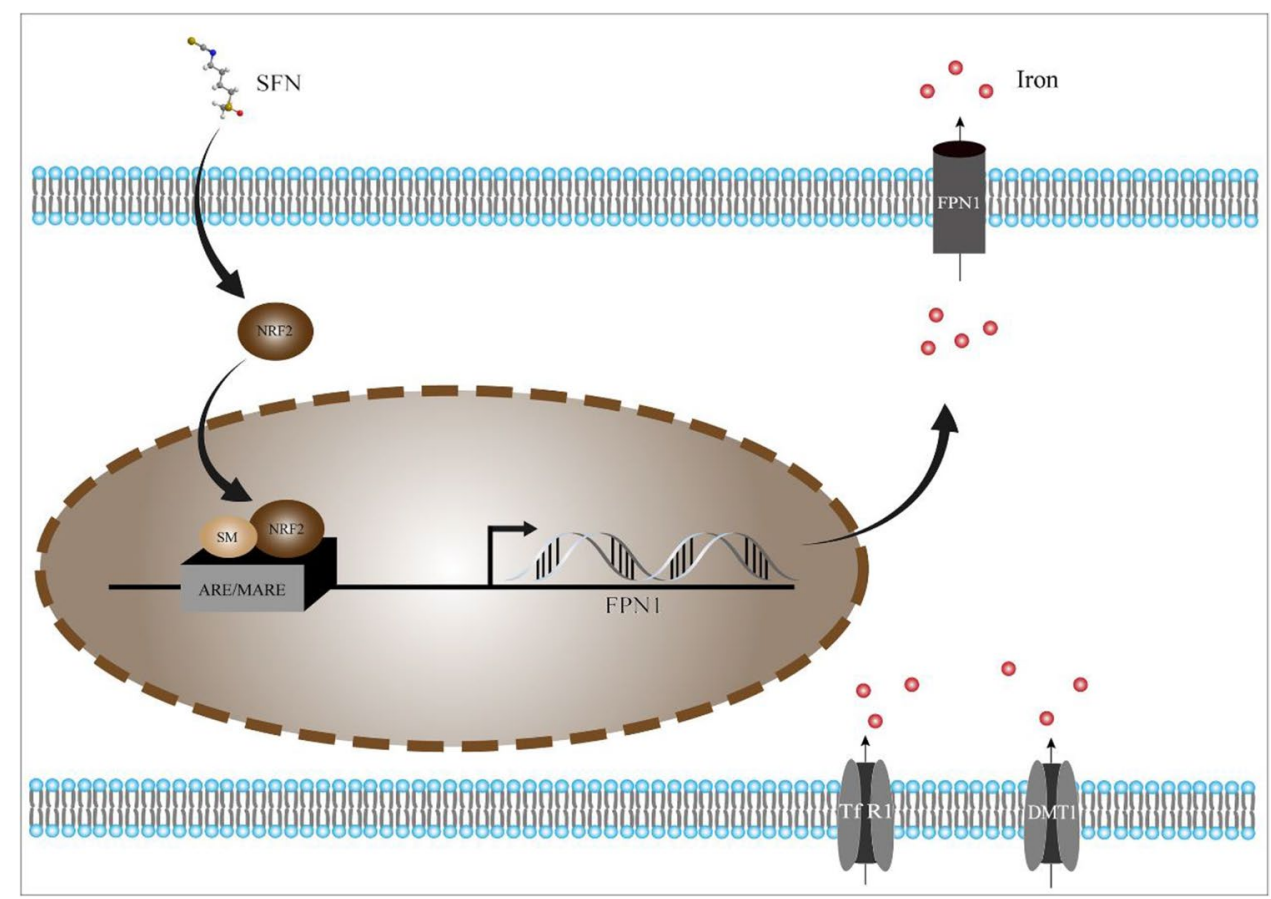


utilized ferroptosis inducer today (Yang et al. 2020). Surprisingly, the administration of erastin prevented the positive effects of SFN and increased the degree of ferroptosis while having no effect on the expression of NRF2 and FPN1, indicating that ferroptosis is controlled by targeting the NRF2/FPN1 signaling pathway. Together, we can conclude that hypo-expression of FPN1 can lead to intracellular iron accumulation and aggravate ferroptosis in the diabetic heart disease, and that SFN further stimulates NRF2 to enhance the transcription of FPN1 and reduce myocardial IRI in diabetic rats.

This study has certain drawbacks. On the one hand, our study is limited to STZ-induced type 1 diabetic rats. Because type 2 diabetes is the most frequent type of diabetes, determining the importance of the NRF2/FPN1 signaling pathway in this illness is crucial. On the other hand, due to the experiment's technical constraints, we were unable to successfully extract NRF2 from the nucleus of the cells. Despite the fact that NRF2's transcriptional action is performed in the nucleus, no research have clearly demonstrated the degree of NRF2 translocation from the cytoplasm to the nucleus, which was one of the key reasons why we decided to extract the total NRF2. Precisely for these reasons, further study is still needed to uncover the underlying molecular mechanism.

In conclusion, our present study demonstrated that NRF2/FPN1 signal pathway is a pivotal mechanism in diabetic myocardial IRI by regulating iron metabolic homeostasis to restrict ferroptosis, and activation of NRF2/FPN1 signal pathway can relieve diabetic myocardial IRI to some extent. Modulating ferroptosis by impacting iron metabolism might provide an effective strategy for the prophylaxis and treatment of diabetic myocardial IRI.

Author contribution HT designed this study, performed major parts of the experiments, and wrote the manuscript. YZ and YHX helped in experimental design. JT and LL participated in in vivo experiments and western blot experiments. YL participated in the interpretation of our results. ZYX and ZQ developed the concept and provided guidance during the study.

Funding This study was supported by grants from the National Natural Science Foundation of China (81970722). The authors would like to thank the Central Laboratory, Renmin Hospital of Wuhan University (Wuhan, Hubei, China) for their support of our study.

\section{Declarations}

Ethics approval International, national and/or institutional rules for animal care and usage were adhered to.

Conflict of interest The authors declare no competing interests.
Open Access This article is licensed under a Creative Commons Attribution 4.0 International License, which permits use, sharing, adaptation, distribution and reproduction in any medium or format, as long as you give appropriate credit to the original author(s) and the source, provide a link to the Creative Commons licence, and indicate if changes were made. The images or other third party material in this article are included in the article's Creative Commons licence, unless indicated otherwise in a credit line to the material. If material is not included in the article's Creative Commons licence and your intended use is not permitted by statutory regulation or exceeds the permitted use, you will need to obtain permission directly from the copyright holder. To view a copy of this licence, visit http://creativecommons.org/licenses/by/4.0/.

\section{References}

Brownlee M (2005) The pathobiology of diabetic complications: a unifying mechanism. Diabetes 54(6):1615-1625. https://doi.org/10. 2337/diabetes.54.6.1615

Crisafulli A, Pagliaro P, Roberto P, Cugusi L, Mercuro G, Lazou A, Beauloye C, Bertrand L, Hausenloy DJ, Aragno M, Penna C (2020) Diabetic cardiomyopathy and ischemic heart disease: prevention and therapy by exercise and conditioning. Int J Mol Sci 21(8):2896. https://doi.org/10.3390/ijms21082896

Davidson SM, Ferdinandy P, Andreadou I, Bøtker HE, Heusch G, Ibáñez B, Ovize M, Schulz R, Yellon DM, Hausenloy DJ, Garcia-Dorado D (2019) Multitarget strategies to reduce myocardial ischemia/reperfusion injury: Jacc review topic of the week. J Am Coll Cardiol 73(1):89-99. https://doi.org/10.1016/j.jacc.2018.09. 086

Dixon SJ, Lemberg KM, Lamprecht MR, Skouta R, Zaitsev EM, Gleason CE, Patel DN, Bauer AJ, Cantley AM, Yang WS, Morrison B 3rd, Stockwell BR (2012) Ferroptosis: an iron-dependent form of nonapoptotic cell death. Cell 149(5):1060-1072. https://doi.org/ 10.1016/j.cell.2012.03.042

Doll S, Freitas FP, Shah R, Aldrovandi M, da Silva MC, Ingold I, Goya Grocin A, Xavier da Silva TN, Panzilius E, Scheel CH, Mourão A, Buday K, Sato M, Wanninger J, Vignane T, Mohana V, Rehberg M, Flatley A, Schepers A, Kurz A, White D, Sauer M, Sattler M, Tate EW, Schmitz W, Schulze A, O'Donnell V, Proneth B, Popowicz GM, Pratt DA, Angeli JPF, Conrad M (2019) Fsp1 is a glutathione-independent ferroptosis suppressor. Nature 575(7784):693-698. https://doi.org/10.1038/s41586-019-1707-0

Donovan A, Lima CA, Pinkus JL, Pinkus GS, Zon LI, Robine S, Andrews NC (2005) The iron exporter ferroportin/Slc40a1 is essential for iron homeostasis. Cell Metab 1(3):191-200. https:// doi.org/10.1016/j.cmet.2005.01.003

Fang X, Wang H, Han D, Xie E, Yang X, Wei J, Gu S, Gao F, Zhu N, Yin X, Cheng Q, Zhang P, Dai W, Chen J, Yang F, Yang HT, Linkermann A, Gu W, Min J, Wang F (2019) Ferroptosis as a target for protection against cardiomyopathy. Proc Natl Acad Sci U S A 116(7):2672-2680. https://doi.org/10.1073/pnas.1821022116

Galluzzi L, Vitale I, Abrams JM, Alnemri ES, Baehrecke EH, Blagosklonny MV, Dawson TM, Dawson VL, El-Deiry WS, Fulda S, Gottlieb E, Green DR, Hengartner MO, Kepp O, Knight RA, Kumar S, Lipton SA, Lu X, Madeo F, Malorni W, Mehlen P, Nuñez G, Peter ME, Piacentini M, Rubinsztein DC, Shi Y, Simon HU, Vandenabeele P, White E, Yuan J, Zhivotovsky B, Melino G, Kroemer G (2012) Molecular definitions of cell death subroutines: recommendations of the nomenclature committee on cell death 2012. Cell Death Differ 19(1):107-120. https://doi.org/10.1038/cdd.2011.96

Gan L, Xie D, Liu J, Bond Lau W, Christopher TA, Lopez B, Zhang L, Gao E, Koch W, Ma XL, Wang Y (2020) Small extracellular microvesicles mediated pathological communications between dysfunctional adipocytes and cardiomyocytes as a novel 
mechanism exacerbating ischemia/reperfusion injury in diabetic mice. Circulation 141(12):968-983. https://doi.org/10.1161/circu lationaha.119.042640

Gozzelino R, Arosio P (2016) Iron homeostasis in health and disease. Int J Mol Sci 17(1):130. https://doi.org/10.3390/ijms17010130

Harada N, Kanayama M, Maruyama A, Yoshida A, Tazumi K, Hosoya T, Mimura J, Toki T, Maher JM, Yamamoto M, Itoh K (2011) Nrf2 regulates ferroportin 1-mediated iron efflux and counteracts lipopolysaccharide-induced ferroportin 1 Mrna suppression in macrophages. Arch Biochem Biophys 508(1):101-109. https:// doi.org/10.1016/j.abb.2011.02.001

Ichihara S (2013) The pathological roles of environmental and redox stresses in cardiovascular diseases. Environ Health Prev Med 18(3):177-184. https://doi.org/10.1007/s12199-012-0326-2

Iwai K (2019) Regulation of cellular iron metabolism: iron-dependent degradation of Irp by Scf(Fbxl5) ubiquitin ligase. Free Radic Biol Med 133:64-68. https://doi.org/10.1016/j.freeradbiomed.2018.09. 011

Kagan VE, Mao G, Qu F, Angeli JP, Doll S, Croix CS, Dar HH, Liu B, Tyurin VA, Ritov VB, Kapralov AA, Amoscato AA, Jiang J, Anthonymuthu T, Mohammadyani D, Yang Q, Proneth B, KleinSeetharaman J, Watkins S, Bahar I, Greenberger J, Mallampalli RK, Stockwell BR, Tyurina YY, Conrad M, Bayır H (2017) Oxidized arachidonic and adrenic pes navigate cells to ferroptosis. Nat Chem Biol 13(1):81-90. https://doi.org/10.1038/nchembio.2238

La Rosa P, Petrillo S, Turchi R, Berardinelli F, Schirinzi T, Vasco G, Lettieri-Barbato D, Fiorenza MT, Bertini ES, Aquilano K, Piemonte F (2021) The Nrf2 induction prevents ferroptosis in Friedreich's ataxia. Redox Biol 38:101791. https://doi.org/10.1016/j.redox.2020. 101791

Lakhal-Littleton S, Wolna M, Carr CA, Miller JJ, Christian HC, Ball V, Santos A, Diaz R, Biggs D, Stillion R, Holdship P, Larner F, Tyler DJ, Clarke K, Davies B, Robbins PA (2015) Cardiac ferroportin regulates cellular iron homeostasis and is important for cardiac function. Proc Natl Acad Sci U S A 112(10):3164-3169. https://doi.org/ $10.1073 /$ pnas. 1422373112

Li W, Li W, Leng Y, Xiong Y, Xia Z (2020) Ferroptosis is involved in diabetes myocardial ischemia/reperfusion injury through endoplasmic reticulum stress. DNA Cell Biol 39(2):210-225. https://doi.org/ 10.1089/dna.2019.5097

Li W, Li W, Wang Y, Leng Y, Xia Z (2021) Inhibition of Dnmt-1 alleviates ferroptosis through Ncoa4 mediated ferritinophagy during diabetes myocardial ischemia/reperfusion injury. Cell Death Discov 7(1):267. https://doi.org/10.1038/s41420-021-00656-0

Liu Z, Han K, Huo X, Yan B, Gao M, Lv X, Yu P, Gao G, Chang YZ (2020a) Nrf2 knockout dysregulates iron metabolism and increases the hemolysis through ros in aging mice. Life Sci 255:117838. https://doi.org/10.1016/j.lfs.2020.117838

Liu Z, Zhang F, Zhao L, Zhang X, Li Y, Liu L (2020b) Protective effect of pravastatin on myocardial ischemia reperfusion injury by regulation of the Mir-93/Nrf2/are signal pathway. Drug Des Devel Ther 14:3853-3864. https://doi.org/10.2147/dddt.S251726

Luo LF, Guan P, Qin LY, Wang JX, Wang N, Ji ES (2021) Astragaloside Iv inhibits adriamycin-induced cardiac ferroptosis by enhancing Nrf2 signaling. Mol Cell Biochem. https://doi.org/10.1007/ s11010-021-04112-6

Ma Q (2010) Transcriptional responses to oxidative stress: pathological and toxicological implications. Pharmacol Ther 125(3):376-393. https://doi.org/10.1016/j.pharmthera.2009.11.004

Manz DH, Blanchette NL, Paul BT, Torti FM, Torti SV (2016) Iron and cancer: recent insights. Ann N Y Acad Sci 1368(1):149-161. https:// doi.org/10.1111/nyas. 13008

Marro S, Chiabrando D, Messana E, Stolte J, Turco E, Tolosano E, Muckenthaler MU (2010) Heme controls ferroportin1 (Fpn1) transcription involving Bach1, Nrf2 and a mare/are sequence motif at position
-7007 of the Fpn1 promoter. Haematologica 95(8):1261-1268. https://doi.org/10.3324/haematol.2009.020123

Moi P, Chan K, Asunis I, Cao A, Kan YW (1994) Isolation of NfE2-related factor 2 (Nrf2), a Nf-E2-like basic leucine zipper transcriptional activator that binds to the tandem Nf-E2/Ap1 repeat of the beta-globin locus control region. Proc Natl Acad Sci U S A 91(21):9926-9930. https://doi.org/10.1073/pnas.91.21.9926

Piao CS, Gao S, Lee GH, Kim DS, Park BH, Chae SW, Chae HJ, Kim SH (2010) Sulforaphane protects ischemic injury of hearts through antioxidant pathway and mitochondrial K(Atp) channels. Pharmacol Res 61(4):342-348. https://doi.org/10.1016/j.phrs.2009.11.009

Qi X, Zhang Y, Guo H, Hai Y, Luo Y, Yue T (2020) Mechanism and intervention measures of iron side effects on the intestine. Crit Rev Food Sci Nutr 60(12):2113-2125. https://doi.org/10.1080/10408 398.2019.1630599

Qiu Z, Lei S, Zhao B, Wu Y, Su W, Liu M, Meng Q, Zhou B, Leng Y, Xia ZY (2017) Nlrp3 inflammasome activation-mediated pyroptosis aggravates myocardial ischemia/reperfusion injury in diabetic rats. Oxid Med Cell Longev 2017:9743280. https://doi.org/10.1155/ 2017/9743280

Qu X, Chen X, Shi Q, Wang X, Wang D, Yang L (2019) Resveratrol alleviates ischemia/reperfusion injury of diabetic myocardium via inducing autophagy. Exp Ther Med 18(4):2719-2725. https://doi. org/10.3892/etm.2019.7846

Tan Y, Ichikawa T, Li J, Si Q, Yang H, Chen X, Goldblatt CS, Meyer CJ, Li X, Cai L, Cui T (2011) Diabetic downregulation of Nrf2 activity via Erk contributes to oxidative stress-induced insulin resistance in cardiac cells in vitro and in vivo. Diabetes 60(2):625-633. https:// doi.org/10.2337/db10-1164

Wang WK, Lu QH, Wang X, Wang B, Wang J, Gong HP, Wang L, Li H, Du YM (2017) Ulinastatin attenuates diabetes-induced cardiac dysfunction by the inhibition of inflammation and apoptosis. Exp Ther Med 14(3):2497-2504. https://doi.org/10.3892/etm.2017.4824

Wang C, Zhu L, Yuan W, Sun L, Xia Z, Zhang Z, Yao W (2020) Diabetes aggravates myocardial ischaemia reperfusion injury via activating Nox2-related programmed cell death in an Ampk-dependent manner. J Cell Mol Med 24(12):6670-6679. https://doi.org/10.1111/ jcmm. 15318

Xiao C, Xia ML, Wang J, Zhou XR, Lou YY, Tang LH, Zhang FJ, Yang JT, Qian LB (2019) Luteolin attenuates cardiac ischemia/reperfusion injury in diabetic rats by modulating Nrf2 antioxidative function. Oxid Med Cell Longev 2019:2719252. https://doi.org/10.1155/ 2019/2719252

Xu G, Zhao X, Fu J, Wang X (2019) Resveratrol increase myocardial Nrf2 expression in type 2 diabetic rats and alleviate myocardial ischemia/reperfusion injury (Miri). Ann Palliat Med 8(5):565-575. https://doi.org/10.21037/apm.2019.11.25

Yang S, Deng Q, Sun L, Dong K, Li Y, Wu S, Huang R (2019) Salmonella effector Spvb interferes with intracellular iron homeostasis via regulation of transcription factor Nrf2. Faseb j 33(12):13450-13464. https://doi.org/10.1096/fj.201900883RR

Yang Y, Luo M, Zhang K, Zhang J, Gao T, Connell DO, Yao F, Mu C, Cai B, Shang Y, Chen W (2020) Nedd4 ubiquitylates Vdac2/3 to suppress erastin-induced ferroptosis in melanoma. Nat Commun 11(1):433. https://doi.org/10.1038/s41467-020-14324-x

Yuan H, Li X, Zhang X, Kang R, Tang D (2016) Identification of Acsl4 as a biomarker and contributor of ferroptosis. Biochem Biophys Res Commun 478(3):1338-1343. https://doi.org/10.1016/j.bbrc. 2016.08.124

Zhang B, Zhai M, Li B, Liu Z, Li K, Jiang L, Zhang M, Yi W, Yang J, Yi D, Liang H, Jin Z, Duan W, Yu S (2018) Honokiol ameliorates myocardial ischemia/reperfusion injury in type 1 diabetic rats by reducing oxidative stress and apoptosis through activating the Sirt1-Nrf2 signaling pathway. Oxid Med Cell Longev 2018:3159801. https:// doi.org/10.1155/2018/3159801 
Zhao D, Yang J, Yang L (2017) Insights for oxidative stress and mtor signaling in myocardial ischemia/reperfusion injury under diabetes. Oxid Med Cell Longev 2017:6437467. https://doi.org/10.1155/ 2017/6437467

Zhou XR, Ru XC, Xiao C, Pan J, Lou YY, Tang LH, Yang JT, Qian LB (2021) Sestrin2 is involved in the Nrf2-regulated antioxidative signaling pathway in luteolin-induced prevention of the diabetic rat heart from ischemia/reperfusion injury. Food Funct 12(8):3562-3571. https://doi.org/10.1039/d0fo02942d

Publisher's note Springer Nature remains neutral with regard to jurisdictional claims in published maps and institutional affiliations. 